\title{
An Efficient and Straightforward Access to Sulfur Substituted [2.2]Paracyclophanes: Application to Stereoselective Sulfenate Salt Alkylation
}

Jean-François Lohier, ${ }^{a}$ Florian Foucoin, ${ }^{a}$ Paul-Alain Jaffrès, ${ }^{b}$ José I. Garcia, ${ }^{c}$ Jana Sopková-de Oliveira Santos, ${ }^{d}$ Stéphane Perrio, ${ }^{a,{ }^{*}}$ and Patrick Metzner ${ }^{a}$

${ }^{a}$ Laboratoire de Chimie Moléculaire et Thio-organique, ENSICAEN,

Université de Caen Basse-Normandie, CNRS, 6 Boulevard du Maréchal Juin, 14050 Caen, France

${ }^{b}$ Laboratoire de Chimie Electrochimie Moléculaire Chimie Analytique,

Université de Bretagne Occidentale, CNRS, 6 Avenue Le Gorgeu, 29238 Brest, France

${ }^{c}$ Departamento de Química Orgánica, Instituto de Ciencia de Materiales de Aragon, Universidad de Zaragoza-CSIC, 50009 Zaragoza, Spain.

${ }^{d}$ Centre d'Etudes et de Recherche sur le Médicament de Normandie,

Université de Caen Basse-Normandie, 5 Rue Vaubénard, 14032 Caen, France

e-mail: stephane.perrio@ensicaen.fr

Supporting Information

General Conditions

General Procedures

Synthesis and Spectral Data

Theoretical Calculations

S10

Crystal Structure Determination of 9a-c

S13

Copies of ${ }^{1} \mathrm{H}$ and ${ }^{13} \mathrm{C}$ Spectra 


\section{General conditions}

Dry THF, $\mathrm{CH}_{2} \mathrm{Cl}_{2}$ and toluene were displayed by a passage down an activated alumina column. All other reagents and solvents were used as received from commercial sources. All reactions were performed in oven-dried glassware, under an atmosphere of dry nitrogen. Reactions were purified by chromatography column with silica gel Si $60(0.040-0.063 \mathrm{~nm})$. Thin layer chromatography was carried out on silica gel $60 \mathrm{~F}_{254}(1.1 \mathrm{~mm})$ with spot detection under UV light or through $\mathrm{I}_{2}$ or $\mathrm{KMnO}_{4}$ oxidation. Melting points were obtained on a capillary apparatus and are uncorrected. NMR spectra were obtained in $\mathrm{CDCl}_{3}$ unless stated otherwise. All chemical shifts $(\delta)$ and coupling constants are quoted in parts per million (ppm) and Hertz (Hz) respectively. The following abbreviations are used to designate the multiplicity of the signals: $\mathrm{s}=$ singlet; $\mathrm{d}=$ doublet $; \mathrm{t}=$ triplet $; \mathrm{m}=$ multiplet; $\mathrm{br}=$ broad; $\mathrm{app}=$ apparent and combinations thereof. The chemical shifts are calibrated to TMS $(\delta \mathrm{H} 0.00)$ or residual proton and carbon resonance of the solvent $\mathrm{CDCl}_{3}\left(\delta \mathrm{H} 7.26\right.$ and $\delta \mathrm{C} 77.16$ ). ${ }^{29} \mathrm{Si}$ chemical shifts are referred to external TMS. IR and mass spectra were recorded on FT-IR and GC/MS/MS instruments, respectively. Only peaks of an intensity $>10 \%$ (except decisive ones) are listed for mass spectra.

( \pm -Trans-3-phenyl-2-(phenylsulfonyl)loxaziridine 11a was prepared from benzaldehyde and benzenesulfonamide via the corresponding $N$-(phenylsulfonyl)imine according to a published procedure. $^{1}( \pm$ )-Trans-3-( $t$-butyl)-3-methyl-2-(phenylsulfonyl)oxaziridine $\mathbf{1 1 b}$ was prepared as previously described by us. ${ }^{2}$ The only modification concerns the oxidation procedure of the intermediate imine, which was carried out using recently developed conditions ${ }^{3}$ with $\mathrm{MCPBA} / \mathrm{KOH}$.

${ }^{1}$ (a) Vishwakarma, L. C.; Stringer, O. D.; Davis, F. A. Org. Synth. 1988, 66, 203-210. (b) Davis F. A.; Chattopadhyay, S.; Towson, J. C.; Lal, S.; Reddy, T. J. Org. Chem. 1988, 53, 20872089.

${ }^{2}$ Sandrinelli, F.; Boudou, C.; Caupène, C.; Averbuch-Pouchot, M.-T.; Perrio, S.; Metzner, P. Synlett 2006, 3289-3293.

${ }^{3}$ García Ruano, J. L.; Alemán, J.; Fajardo, C.; Parra, A. Org. Lett. 2005, 7, 5493-5496. 


\section{General Procedures}

Oxidation of Sulfides into Sulfoxides with $\mathrm{H}_{2} \mathrm{O}_{2} / \mathbf{F}_{3} \mathrm{CCH}_{2} \mathrm{OH}$ System. To a $1 \mathrm{M}$ solution of sulfide in 2,2,2-trifluoroethanol, cooled to $0{ }^{\circ} \mathrm{C}, \mathrm{H}_{2} \mathrm{O}_{2}\left(1.1\right.$ equiv., $30 \%$ solution in $\mathrm{H}_{2} \mathrm{O}$ ) was added dropwise. The reaction mixture was allowed to warm to room temperature and stirred for 20 h. Solid sodium sulfite (2.1 equiv.) was added then the reaction mixture was heated at $50{ }^{\circ} \mathrm{C}$ for $30 \mathrm{~min}$. The resulting mixture was filtered on celite, dried over $\mathrm{MgSO}_{4}$, filtered and evaporated under reduced pressure. The crude product was then purified by column chromatography on silica gel to afford the anticipated sulfoxide.

Oxidation of Sulfides into Sulfoxides with Oxaziridines 11. To a $0.5 \mathrm{M}$ solution of sulfide in $\mathrm{CH}_{2} \mathrm{Cl}_{2}$, cooled to $0{ }^{\circ} \mathrm{C}$, oxaziridine 11 (1.1 equiv.) dissolved in $\mathrm{CH}_{2} \mathrm{Cl}_{2}(0.5 \mathrm{M}$ concentration) was added dropwise. The reaction mixture was allowed to warm slowly to room temperature and stirred until the reaction is completed according to TLC. Solvent was removed under reduced pressure then the crude was purified by column chromatography on silica gel to give the desired sulfoxide.

Conversion of $\beta$-Sulfanyl Ester 4 into Sulfides 3. A solution of 4 ( 1 equiv.) in dry THF was cooled to $-78{ }^{\circ} \mathrm{C}$ or $-45^{\circ} \mathrm{C}$ and $t$-BuOK (commercial $1 \mathrm{M}$ solution in THF, 1.1 eq) was added. After stirring at the same temperature for $20 \mathrm{~min}$, the alkyl halide (1.2 mmol, 1.2 equiv.) was added. The reaction mixture was allowed to warm to room temperature. After addition of $\mathrm{H}_{2} \mathrm{O}$, concentration in vacuum, extraction with EtOAc $(15 \mathrm{~mL})$, the organic layer was washed with $\mathrm{H}_{2} \mathrm{O}(15 \mathrm{~mL})$. The aqueous layer was extracted with $\mathrm{CH}_{2} \mathrm{Cl}_{2}(2515 \mathrm{~mL})$. The organic fractions were combined, dried over $\mathrm{MgSO}_{4}$, filtered and evaporated to dryness. The resulting crude product was then purified by column chromatography on silica gel to afford the anticipated sulfide 3 .

Sulfenate Generation from $\beta$-Sulfinyl Ester 5 and Conversion into Sulfoxides 9. A solution of 5 (1 equiv.) and alkyl halide (1.1 equiv.) in dry THF was cooled to $-78{ }^{\circ} \mathrm{C}$ and $t$ BuOK ( $1 \mathrm{M}$ solution in THF, 1.1 equiv.) was added dropwise. After stirring at $-78{ }^{\circ} \mathrm{C}$ for $30 \mathrm{~min}$, $\mathrm{H}_{2} \mathrm{O}$ was added and the reaction mixture was allowed to warm to room temperature. Volatiles were removed under reduced pressure and the aqueous phase was extracted twice with $\mathrm{CH}_{2} \mathrm{Cl}_{2}$. The collected organic layers were dried over $\mathrm{MgSO}_{4}$ and concentrated under vacuum. The residue was analysed by ${ }^{1} \mathrm{H}$ NMR and purified by column chromatography on silica gel to furnish the anticipated sulfoxides 9 . 
3-[(2-Carboxymethyl)ethylsulfanyl]propionic Acid Methyl Ester (12). ${ }^{4}$ Methyl acrylate $(1 \mathrm{~mL}, 11 \mathrm{mmol})$ was added to a solution of 3-sulfanylpropanoic acid methyl ester $(1.1 \mathrm{~mL}, 10$ mmol) in $\mathrm{H}_{2} \mathrm{O}(20 \mathrm{~mL})$. The reaction mixture was heated at $35{ }^{\circ} \mathrm{C}$ for $30 \mathrm{~min}$, cooled to room temperature and extracted with $\mathrm{CH}_{2} \mathrm{Cl}_{2}\left(2530 \mathrm{~mL}\right.$ ). The organic layer was dried over $\mathrm{MgSO}_{4}$, filtered and concentrated under reduced pressure to furnish the anticipated thioether $\mathbf{1 2}$ as a colourless oil (2.06 g, $10 \mathrm{mmol}$, quantitative). The product was used in the next step without further purification. TLC $\left(\mathrm{CH}_{2} \mathrm{Cl}_{2},\right) R_{f}=0.18 .{ }^{1} \mathrm{H} \mathrm{NMR}\left(\mathrm{CDCl}_{3}, 250 \mathrm{MHz}\right) \delta: 2.58-2.64(\mathrm{~m}, 4 \mathrm{H})$, $2.77-2.83(\mathrm{~m}, 4 \mathrm{H}), 3.69(\mathrm{~s}, 6 \mathrm{H}) .{ }^{13} \mathrm{C} \mathrm{NMR}\left(\mathrm{CDCl}_{3}, 63 \mathrm{MHz}\right) \delta: 27.0,34.6,51.8,172.3$. IR $(\mathrm{KBr}$, $\left.\mathrm{cm}^{-1}\right) v: 1730(\mathrm{C}=\mathrm{O}), 1436,1356,1243,1194,1170,1141 . \mathrm{MS}(\mathrm{ESI}): \mathrm{m} / z(\%) 207\left[(\mathrm{M}+\mathrm{H})^{+}\right.$, 79], 193 (63), 175 (100), 143 (28). HRMS (ESI) Calcd for $\mathrm{C}_{8} \mathrm{H}_{15} \mathrm{O}_{4} \mathrm{~S}$ : 207.0691, Found: 207.0690.

3-[(2-Carboxymethyl)ethylsulfinyl]propionic Acid Methyl Ester (7). ${ }^{5}$ A solution of thioether $12(2.06 \mathrm{~g}, 10 \mathrm{mmol})$ in a mixture of acetone $(50 \mathrm{~mL})$ and $\mathrm{H}_{2} \mathrm{O}(50 \mathrm{~mL})$ was cooled to 0 ${ }^{\circ} \mathrm{C} . \mathrm{NaIO}_{4}(2.14 \mathrm{~g}, 10 \mathrm{mmol})$ was added portionwise and the reaction mixture was stirred at room temperature for $24 \mathrm{~h}$. Liberated $\mathrm{NaIO}_{3}$ was filtered, volatiles were removed under vacuum and the residue was extracted with $\mathrm{CH}_{2} \mathrm{Cl}_{2}\left(2530 \mathrm{~mL}\right.$ ). After drying over $\mathrm{MgSO}_{4}$, filtration and concentration, purification by chromatography on silica gel $\left(\mathrm{MeOH} / \mathrm{CH}_{2} \mathrm{Cl}_{2}, 5: 95\right)$ afforded the desired sulfoxide 7 as a white solid (2.07 g, $0.93 \mathrm{mmol}, 93 \%)$. TLC $\left(\mathrm{MeOH} / \mathrm{CH}_{2} \mathrm{Cl}_{2}, 5: 95\right) R f=$ 0.73. mp: $65{ }^{\circ} \mathrm{C}\left(\mathrm{AcOEt} /\right.$ pentane) $\left(\right.$ lit. $\left.^{5} \mathrm{mp} 66{ }^{\circ} \mathrm{C}\right) .{ }^{1} \mathrm{H} \mathrm{NMR}\left(\mathrm{CDCl}_{3}, 250 \mathrm{MHz}\right) \delta: 2.81-3.13$ (m, $8 \mathrm{H}), 3.72(\mathrm{~s}, 6 \mathrm{H}) .{ }^{13} \mathrm{C} \mathrm{NMR}\left(\mathrm{CDCl}_{3}, 63 \mathrm{MHz}\right) \delta: 26.8,47.0,52.1,171.5 . \mathrm{IR}\left(\mathrm{KBr}, \mathrm{cm}^{-1}\right)$ v: 1731 $(\mathrm{C}=\mathrm{O}), 1361,1231,1166,1024,988,968 . \mathrm{MS}(\mathrm{ESI}): \mathrm{m} / z(\%) 245\left[(\mathrm{M}+\mathrm{Na})^{+}, 100\right], 223[(\mathrm{M}+$ $\left.\mathrm{H})^{+}, 64\right], 191$ (25), 159 (16), 137 (18), 119 (22).

3-\{[2.2]Paracyclophan-4-yl\}sulfanylpropionic Acid Methyl Ester (4). Sulfinyl diester 7 (289 $\mathrm{mg}, 1.3 \mathrm{mmol}$ ) was added to a solution of [2.2]paracyclophane 1 (208 $\mathrm{mg}, 1.0 \mathrm{mmol})$ in $\mathrm{CH}_{2} \mathrm{Cl}_{2}(8 \mathrm{~mL})$. The reaction mixture was cooled to $-40{ }^{\circ} \mathrm{C}$ and triflic anhydride $(250 \mu \mathrm{L}, 1.49$ mmol) was added dropwise. After stirring successively at $-35^{\circ} \mathrm{C}$ for $20 \mathrm{~min}, 0{ }^{\circ} \mathrm{C}$ for 30 min and room temperature for $16 \mathrm{~h}$, solvent was removed under reduced pressure. The oily residue was dissolved in THF $(2 \mathrm{~mL})$ and $\operatorname{dry~}_{3} \mathrm{Et}_{3} \mathrm{~N}(5 \mathrm{~mL})$ was added. The biphasic system was stirred vigorously at room temperature for $12 \mathrm{~h}$. Volatiles were eliminated under reduced pressure and purification by chromatography on silica gel (pentane/ $\mathrm{CH}_{2} \mathrm{Cl}_{2}, 1: 1$ ) afforded the title sulfide 4 as a white solid (246 mg, $0.75 \mathrm{mmol}, 75 \%)$. TLC $\left(\mathrm{CH}_{2} \mathrm{Cl}_{2} /\right.$ pentane, 1:1) $R_{f}=0.49 . \mathrm{mp}: 48{ }^{\circ} \mathrm{C} .{ }^{1} \mathrm{H}$ NMR $\left(\mathrm{CDCl}_{3}, 250 \mathrm{MHz}\right) \delta: 2.53\left(\mathrm{t}_{\mathrm{app}}, 2 \mathrm{H}, J=7.5\right), 2.74-2.86(\mathrm{~m}, 1 \mathrm{H}), 2.92-3.26(\mathrm{~m}, 6 \mathrm{H}), 2.98$ (t, 2H, $J=7.5), 3.53(\mathrm{ddd}, 1 \mathrm{H}, J=2.5,10.0$ and 12.6), 3.67 (s, 3H), 6.37-6.55 (m, 6H), 6.91 (dd, $1 \mathrm{H}, J=1.8$ and 7.8$) .{ }^{13} \mathrm{C} \mathrm{NMR}\left(\mathrm{CDCl}_{3}, 63 \mathrm{MHz}\right) \delta: 29.1,34.0,34.49,34.54,35.1,35.5,51.9$, 129.2 , 131.6, 132.1, 133.0, 133.4, 134.9, 135.7, 136.2, 139.3, 139.6, 140.5, 140.8, 172.5. IR $\left(\mathrm{KBr}, \mathrm{cm}^{-1}\right)$ v: 2923, $1740(\mathrm{C}=\mathrm{O}), 1437,1356,1242,1193,1165,1059,848,717 . \mathrm{MS}(\mathrm{EI}): \mathrm{m} / \mathrm{z}$ (\%) $326\left(\mathrm{M}^{+}, 100\right), 252$ (42), 222 (52), 163 (83), 147 (21), 104 (21), 91 (20), 78 (15). Anal. calcd for $\mathrm{C}_{20} \mathrm{H}_{22} \mathrm{O}_{2} \mathrm{~S}$ : C 73.58, H 6.79, S 9.82; Found: C 73.86, H 6.83, S 9.64.

\footnotetext{
${ }^{4}$ Gershbein, L.; Hurd, C. J. Am. Chem. Soc. 1947, 69, 241-242.

${ }^{5}$ Armstrong, S. J. Chem. Soc. B, 1971, 1747-1751.
} 
[2.2]Paracyclophane-4-thiol (2). ${ }^{6}$ A solution of $\beta$-sulfanyl ester 4 (246 mg, $0.75 \mathrm{mmol}$ ) in dry THF ( $4 \mathrm{~mL}$ ) was cooled to $-78^{\circ} \mathrm{C}$ and $t$-BuOK ( $830 \mu \mathrm{L}$ of a $1 \mathrm{M}$ solution in THF) was added dropwise. After stirring at $-78{ }^{\circ} \mathrm{C}$ for $30 \mathrm{~min}$, the reaction mixture was poured into a $\mathrm{H}_{2} \mathrm{O}$ /ice mixture $(5 \mathrm{~mL})$ and aqueous $1 \mathrm{M} \mathrm{HCl}$ solution was added until reaching $\mathrm{pH} 1$. The resulting precipitate was rinsed with $\mathrm{H}_{2} \mathrm{O}$ and dried under high vacuum to give the title mercaptan 2 (160 mg, $0.67 \mathrm{mmol}, 89 \%$ ) as a white solid. TLC (pentane/ $\left.\mathrm{CH}_{2} \mathrm{Cl}_{2}, 9: 1\right) R_{f}=0.53 . \mathrm{mp}: 140{ }^{\circ} \mathrm{C}$ (lit. $\left.{ }^{6} \mathrm{mp} 140-141{ }^{\circ} \mathrm{C}\right) .{ }^{1} \mathrm{H}$ NMR $\left(\mathrm{CDCl}_{3}, 250 \mathrm{MHz}\right) \delta$ : 2.75-3.45 (m, 8H), $6.21(\mathrm{~d}, 1 \mathrm{H}, J=1.3$ ), 6.37-6.43 (m, 3H), 6.47 (dd, $1 \mathrm{H}, J=1.3$ and 7.8), 6.57 (dd, $1 \mathrm{H}, J=1.8$ and 7.8), $7.21(\mathrm{dd}, 1 \mathrm{H}, J$ $=1.8$ and 7.9) ${ }^{13} \mathrm{C} \mathrm{NMR}\left(\mathrm{CDCl}_{3}, 63 \mathrm{MHz}\right) \delta: 33.2,34.8,35.0,35.5,127.8,130.6,131.7,132.1$, 132.9, 133.5, 134.9, 135.7, 138.6, 139.3, 139.4, 140.5. IR $\left(\mathrm{KBr}, \mathrm{cm}^{-1}\right)$ v: 2925, 1059, 897, 849,

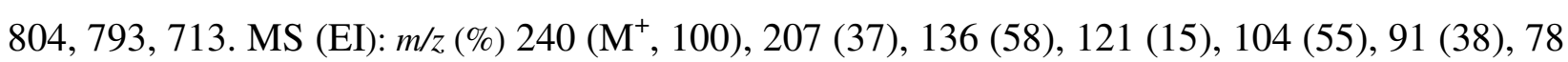
(25).

4-Benzylsulfanyl[2.2]paracyclophane (3a). Obtained following the general procedure described at $-45^{\circ} \mathrm{C}$ and using $\beta$-sulfanyl ester 4 (1.15 g, $\left.3.53 \mathrm{mmol}\right)$ in dry THF $(15 \mathrm{~mL}), t$ BuOK $(3.9 \mathrm{~mL})$ and benzyl bromide $(465 \mu \mathrm{L}, 3.89 \mathrm{mmol})$ as the electrophile. Purification by chromatography $\left(\mathrm{CH}_{2} \mathrm{Cl}_{2} /\right.$ pentane, 1:9) afforded the title benzyl sulfide 3a as a white solid (987 $\mathrm{mg}, 2.99 \mathrm{mmol}, 85 \%)$. TLC $\left(\mathrm{CH}_{2} \mathrm{Cl}_{2} /\right.$ pentane, 1:4) $R_{f}=0.25 . \mathrm{mp}: 108{ }^{\circ} \mathrm{C} .{ }^{1} \mathrm{H} \mathrm{NMR}\left(\mathrm{CDCl}_{3}, 400\right.$ MHz) $\delta: 2.76(\mathrm{ddd}, 1 \mathrm{H}, J=5.8,10.5$ and 13.3), 2.88-2.95 (m, 1H), 3.01-3.09 (m, 4H), 3.23 (ddd, $1 \mathrm{H}, J=5.8,10.1$ and 13.0), 3.48 (ddd, $1 \mathrm{H}, J=2.2,10.1$ and 12.8), 3.94 (s, 2H), 6.27 (dd, $1 \mathrm{H}, J=$ 1.9 and 7.8), $6.32(\mathrm{~d}, 1 \mathrm{H}, J=1.3), 6.41-6.47(\mathrm{~m}, 3 \mathrm{H}), 6.52(\mathrm{dd}, 1 \mathrm{H}, J=1.9$ and 7.8), $6.95(\mathrm{dd}$, $1 \mathrm{H}, J=7.8), 7.22-7.32(\mathrm{~m}, 5 \mathrm{H}) .{ }^{13} \mathrm{C} \mathrm{NMR}\left(\mathrm{CDCl}_{3}, 63 \mathrm{MHz}\right) \delta: 33.9,34.4,35.1,35.5,38.7$, 127.3, 128.6, 129.0, 129.1, 131.0, 132.0, 132.9, 133.4, 134.4, 134.7, 137.6, 137.7, 139.3, 139.6, 139.8, 140.4. IR (KBr, cm $\left.{ }^{-1}\right)$ v: 2924, 1058, 893, 848, 797, 717, 694. MS (EI): $\mathrm{m} / z(\%) 330\left(\mathrm{M}^{+}\right.$, 100), 315 (17), 225 (15), 211 (12), 193 (40), 135 (28), 91 (64), 65 (18). Anal. calcd for $\mathrm{C}_{23} \mathrm{H}_{22} \mathrm{~S}$ : C 83.59, H 6.71, S 9.70; Found: C 83.19, H 6.79, S 10.02.

4-Methylsulfanyl[2.2]paracyclophane (3b). Obtained following the general procedure described at $-78{ }^{\circ} \mathrm{C}$ and using $\beta$-sulfanyl ester $4(331 \mathrm{mg}, 1.02 \mathrm{mmol})$ in dry THF $(5 \mathrm{~mL}), t$ $\operatorname{BuOK}(1.12 \mathrm{~mL})$ and methyl iodide $(70 \mu \mathrm{L}, 1.12 \mathrm{mmol})$ as the electrophile. Purification by chromatography $\left(\mathrm{CH}_{2} \mathrm{Cl}_{2} /\right.$ pentane, 1:9) afforded the title methyl sulfide $\mathbf{3 b}$ as a white solid (242 $\mathrm{mg}, 0.95 \mathrm{mmol}, 93 \%)$. TLC $\left(\mathrm{CH}_{2} \mathrm{Cl}_{2} /\right.$ pentane, 1:9) $R f=0.49 . \mathrm{mp}: 96{ }^{\circ} \mathrm{C} .{ }^{1} \mathrm{H} \mathrm{NMR}\left(\mathrm{CDCl}_{3}, 250\right.$ MHz) $\delta: 2.35$ (s, 3H), 2.78 (ddd, $1 \mathrm{H}, J=5.8,10.5$ and 13.0), 2.94-3.11 (m, 5H), 3.26 (ddd, $1 \mathrm{H}, J$ $=5.8,10.0$ and 12.8), $3.45(\mathrm{ddd}, 1 \mathrm{H}, J=2.0,10.0$ and 13.0), $6.10(\mathrm{~s}, 1 \mathrm{H}), 6.34-6.41(\mathrm{~m}, 3 \mathrm{H})$, $6.46\left(\mathrm{dd}, 1 \mathrm{H}, J=1.7\right.$ and 7.8), $6.55\left(\mathrm{dd}, 1 \mathrm{H}, J=1.9\right.$ and 7.8), $7.05(\mathrm{dd}, 1 \mathrm{H}, J=1.8$ and 7.8$) .{ }^{13} \mathrm{C}$ NMR $\left(\mathrm{CDCl}_{3}, 63 \mathrm{MHz}\right) \delta: 15.3,33.4,34.1,35.3,35.5,128.1,129.5,130.5,131.6,132.9,133.6$, 134.6, 137.9, 139.1, 139.3, 139.5, 140.5. IR $\left(\mathrm{KBr}, \mathrm{cm}^{-1}\right)$ v: 2926, 1437, 1058, 911, 891, 844, 792, 716. MS (EI): $m / z$ (\%) $254\left(\mathrm{M}^{+}, 100\right), 150$ (91), 135 (24), 104 (48), 91 (13), 78 (18). Anal. calcd for $\mathrm{C}_{17} \mathrm{H}_{18} \mathrm{~S}$ : C 80.26, H 7.13; Found: C 79.97, H 7.34.

${ }^{6}$ Hopf, H.; Kane, V.; Gerdes, A.; Grahn, W.; Ernst, L.; Dix, I.; Jones, P. Tetrahedron Lett. 2001, 42, 373-376. 
4-Ethylsulfanyl[2.2]paracyclophane (3c). Obtained following the general procedure described at $-78{ }^{\circ} \mathrm{C}$ and using $\beta$-sulfanyl ester $4(331 \mathrm{mg}, 1.02 \mathrm{mmol})$ in dry THF (5 mL), $t$ BuOK $(1.12 \mathrm{~mL})$ and ethyl iodide $(90 \mu \mathrm{L}, 1.12 \mathrm{mmol})$ as the electrophile. Purification by chromatography $\left(\mathrm{CH}_{2} \mathrm{Cl}_{2} /\right.$ pentane, 1:9) afforded the title ethyl sulfide $3 \mathbf{c}$ as a white solid (235 $\mathrm{mg}, 0.88 \mathrm{mmol}, 86 \%)$. TLC $\left(\mathrm{CH}_{2} \mathrm{Cl}_{2} /\right.$ pentane, 1:9) $R f=0.50 . \mathrm{mp}: 85{ }^{\circ} \mathrm{C} .{ }^{1} \mathrm{H} \mathrm{NMR}\left(\mathrm{CDCl}_{3}, 250\right.$ MHz) $\delta: 1.31(\mathrm{t}, 3 \mathrm{H}, J=7.5), 2.79-3.16(\mathrm{~m}, 6 \mathrm{H}), 2.80(\mathrm{q}, 2 \mathrm{H}, J=7.5), 3.27(\mathrm{ddd}, 1 \mathrm{H}, J=5.8$, 10.0 and 12.9), 3.54 (ddd, $1 \mathrm{H}, J=2.1,10.0$ and 12.5), $6.30(\mathrm{~s}, 1 \mathrm{H}), 6.39-6.45(\mathrm{~m}, 3 \mathrm{H}), 6.50$ (dd, $1 \mathrm{H}, J=1.7$ and 7.9$), 6.58(\mathrm{dd}, 1 \mathrm{H}, J=1.7$ and 7.8$), 7.04(\mathrm{dd}, 1 \mathrm{H}, \mathrm{J}=1.7$ and 7.8$) .{ }^{13} \mathrm{C} \mathrm{NMR}$ $\left(\mathrm{CDCl}_{3}, 63 \mathrm{MHz}\right) \delta: 14.6,27.5,33.7,34.4,35.2,35.5,128.7,130.4,131.9,132.9,133.4,133.8$, 134.7, 137.7, 139.2, 139.5, 139.6, 140.3. IR (KBr, $\left.\mathrm{cm}^{-1}\right)$ v: 2925, 1471, 1060, 892, 847, 800, 791, 716, 703. MS (EI): m/z (\%) $268\left(\mathrm{M}^{+}, 100\right), 164$ (44), 149 (21), 130 (25), 118 (14), 104 (19), 91 (15). Anal. calcd for $\mathrm{C}_{18} \mathrm{H}_{20} \mathrm{~S}$ : C 80.54, H 7.51, S 11.95; Found: C 80.16, H 7.67, S 12.17.

3-\{[2.2]Paracyclophan-4-yl\}sulfinylpropionic Acid Methyl Ester (5). Obtained following the $\mathrm{H}_{2} \mathrm{O}_{2}$ procedure described and using sulfide 4 (332 mg, $1.02 \mathrm{mmol}$ ) in 2,2,2trifluoroethanol (1 mL) and $\mathrm{H}_{2} \mathrm{O}_{2}(114 \mu \mathrm{L}, 1.12 \mathrm{mmol}, 30 \%$ solution). Purification by chromatography (EtOAc/ $\left.\mathrm{CH}_{2} \mathrm{Cl}_{2}, 1: 1\right)$ afforded sulfoxide $\mathbf{5}$ as an inseparable mixture (3:2 ratio) of diastereoisomers (335 mg, $0.98 \mathrm{mmol}, 96 \%$ ). According to NMR data with a characteristic signal of one diastereotopic $\mathrm{H} 2$, the major configuration was assigned as $\left(S_{\mathrm{p}}, R_{\mathrm{S}}\right)^{*}$. White solid. TLC (EtOAc/ $\left.\mathrm{CH}_{2} \mathrm{Cl}_{2}, 1: 4\right) R_{f}=0.30 . \mathrm{mp}: 71{ }^{\circ} \mathrm{C} .{ }^{1} \mathrm{H} \mathrm{NMR}\left(\mathrm{CDCl}_{3}, 250 \mathrm{MHz}\right) \delta: 2.34-2.44(\mathrm{~m}$, 0.4H, minor), 2.53-3.43 (m, 11H), 3.64 (s, 1.2H, minor), 3.67 (s, 1.8H, major), 4.19 (ddd, 0.6H, $J=2.1,10.4$ and 12.4, major), 6.46-6.69 (m, 5.6H), 6.82-6.87 (m, 1H), 6.95 (d, 0.4H, $J=1.8$, minor). ${ }^{13} \mathrm{C} \mathrm{NMR}\left(\mathrm{CDCl}_{3}, 63 \mathrm{MHz}\right) \delta$ (major): 27.4, 33.2, 35.0, 35.2, 35.9, 49.6, 52.1, 131.6, 132.6, 132.71, 132.73, 133.2, 135.9, 136.4, 138.0, 139.2, 140.1, 140.9, 141.1, 171.7. ${ }^{13} \mathrm{C}$ NMR $\left(\mathrm{CDCl}_{3}, 63 \mathrm{MHz}\right) \delta$ (minor): 25.9, 32.5, 34.5, 35.16, 35.24, 49.5, 52.1, 128.5, 131.0, 132.9, 133.1, 133.2, 135.4, 136.0, 138.9, 139.2, 139.6, 141.9, 142.2, 171.9. IR (KBr, cm $\left.{ }^{-1}\right)$ v: 2928, $1733(\mathrm{C}=\mathrm{O}), 1435,1220,1177,1057,1029,848,733,718 . \mathrm{MS}(\mathrm{ESI}): m / z(\%) 707\left[(2 \mathrm{M}+\mathrm{Na})^{+}\right.$, 100], $\left.685\left[(2 \mathrm{M}+\mathrm{H})^{+}, 8\right], 549(15), 533(26), 365\left[(\mathrm{M}+\mathrm{Na})^{+}, 24\right)\right], 343\left[(\mathrm{M}+\mathrm{H})^{+}, 71\right]$. Anal. calcd for $\mathrm{C}_{20} \mathrm{H}_{22} \mathrm{O}_{3} \mathrm{~S}$ : C 70.15, H 6.48, S 9.36; Found: C 69.84, H 6.62, S 9.46.

1-\{[2.2]Paracyclophan-4-yl\}sulfanyl-2-trimethylsilylethane (13). A mixture of [2.2]paracyclophane-4-thiol $2(314 \mathrm{mg}, 1.31 \mathrm{mmol})$, vinyltrimethylsilane (2 mL, $13.8 \mathrm{mmol})$ and AIBN ( $2 \mathrm{mg}, 0.012 \mathrm{mmol}$ ) was heated at $100{ }^{\circ} \mathrm{C}$ in a sealed tube for $16 \mathrm{~h}$. After cooling to room temperature, addition of $\mathrm{Et}_{2} \mathrm{O}$ and concentration under reduced pressure, the residue was purified by chromatography on silica gel (pentane/ $\mathrm{CH}_{2} \mathrm{Cl}_{2}, 9: 1$ ) to afford sulfide $\mathbf{1 3}$ as a colourless oil (398 mg, $1.17 \mathrm{mmol}, 89 \%)$. TLC $\left(\mathrm{CH}_{2} \mathrm{Cl}_{2} /\right.$ pentane, $\left.1: 9\right) R_{f}=0.38 .{ }^{1} \mathrm{H} \mathrm{NMR}\left(\mathrm{CDCl}_{3}, 250 \mathrm{MHz}\right)$ $\delta: 0.03(\mathrm{~s}, 9 \mathrm{H}), 0.86-0.93(\mathrm{~m}, 2 \mathrm{H}), 2.72-2.84(\mathrm{~m}, 3 \mathrm{H}), 2.88-3.12(\mathrm{~m}, 5 \mathrm{H}), 3.22$ (ddd, 1H, $J=6.0$, 10.0 and 12.9), $3.50(\mathrm{ddd}, 1 \mathrm{H}, J=2.2,10.1$ and 12.8), $6.26(\mathrm{~s}, 1 \mathrm{H}), 6.37(\mathrm{dd}, 1 \mathrm{H}, J=1.8$ and 7.8), $6.41\left(\mathrm{~s}_{\mathrm{app}}, 2 \mathrm{H}\right), 6.46(\mathrm{dd}, 1 \mathrm{H}, J=1.8$ and 7.8), $6.55(\mathrm{dd}, 1 \mathrm{H}, J=1.8$ and 7.8), $6.99(\mathrm{dd}, 1 \mathrm{H}, J$ $=1.8$ and 7.8). ${ }^{13} \mathrm{C} \mathrm{NMR}\left(\mathrm{CDCl}_{3}, 63 \mathrm{MHz}\right) \delta:-1.6,17.2,29.4,33.8,34.4,35.2,35.5,128.8$, $130.4,131.9,132.9,133.4,134.0,134.7,138.3,139.3,139.56,139.62,140.3 .{ }^{29} \mathrm{Si} \mathrm{NMR}\left(\mathrm{CDCl}_{3}\right.$, $79 \mathrm{MHz}) \delta:$ 1.74. IR (KBr, cm $\left.{ }^{-1}\right)$ v: 2951, 2922, 2853, 1456, 1249, 857, 840, 718. MS (ESI): $\mathrm{m} / z$ 
(\%) 341 (46) $\left[\mathrm{M}+\mathrm{H}^{+}\right], 313$ (100). Anal. calcd for $\mathrm{C}_{21} \mathrm{H}_{28} \mathrm{SSi}$ : C 74.05, H 8.29, S 9.41. Found: $\mathrm{C}$ 73.84, H 8.39, S 9.42.

1-\{[2.2]Paracyclophan-4-yl\}sulfinyl-2-trimethylsilylethane (6). Obtained following the $\mathrm{H}_{2} \mathrm{O}_{2}$ procedure described and using sulfide $\mathbf{1 3}$ (340 $\mathrm{mg}, 1.02 \mathrm{mmol}$ ) in 2,2,2-trifluoroethanol (1 $\mathrm{mL})$ and $\mathrm{H}_{2} \mathrm{O}_{2}(105 \mu \mathrm{L}, 1.03 \mathrm{mmol}, 30 \%$ solution $)$. Purification by chromatography (EtOAc/pentane, 1:9) afforded the title sulfoxide 6 (7:3 diastereoisomeric ratio) as a yellow pale solid (320 mg, $0.90 \mathrm{mmol}, 88 \%$ ). The two sulfoxide diastereoisomers could be easily separated according to $R_{f}$ values. TLC (EtOAc/pentane, $\left.1: 9\right) R_{f}=0.14$ and 0.23 . However, as the chirality on the sulphur centre is lost during the next step consisting in the sulfenate generation, isolation of the individual diastereoisomers was not necessary. According to NMR data with a characteristic signal of one diastereotopic $\mathrm{H} 2$, the major configuration was assigned as $\left(S_{\mathrm{p}}, R_{\mathrm{S}}\right)^{*}$. $\mathrm{mp}: 61{ }^{\circ} \mathrm{C} .{ }^{1} \mathrm{H} \mathrm{NMR}\left(\mathrm{CDCl}_{3}, 400 \mathrm{MHz}\right) \delta:-0.07$ (s, 2.7H, minor), -0.03 (s, 6.3H, major), 0.47 (dt, $0.7 \mathrm{H}, J=4.3$ and 13.9, major), 0.63 (dt, $0.3 \mathrm{H}, J=4.2$ and 13.9, minor), 0.81 (dt, $0.3 \mathrm{H}, J=$ 4.2 and 13.9, minor), 0.86 (dt, $0.7 \mathrm{H}, J=4.2$ and 13.9, major), 2.49 (dt, $0.3 \mathrm{H}, J=4.2$ and 13.8, minor), 2.65-2.74 (m, 1H, major + minor), 2.83-3.28 (m, 7H, major + minor), 3.32-3.39 (m, $1 \mathrm{H}$, major + minor $), 4.18(\mathrm{ddd}, 0.7 \mathrm{H}, J=2.3,10.5$ and 12.7 , major $), 6.46-6.66(\mathrm{~m}, 5.7 \mathrm{H}$, major + minor $), 6.84-6.90(\mathrm{~m}, 1 \mathrm{H}$, major + minor $), 6.96(\mathrm{~d}, 0.3 \mathrm{H}, J=1.9$, minor $) .{ }^{13} \mathrm{C} \mathrm{NMR}\left(\mathrm{CDCl}_{3}\right.$, $63 \mathrm{MHz}$ ) $\delta$ (major) : -1.8, 9.7, 33.2, 35.0, 35.33, 35.9, 51.2, 132.5, 132.6, 132.7, 132.8, 133.1, 133.6, 136.2, 138.0, 139.2, 140.3, 140.6, 141.0. ${ }^{13} \mathrm{C} \mathrm{NMR}\left(\mathrm{CDCl}_{3}, 63 \mathrm{MHz}\right) \delta$ (minor): $-1.8,8.0$, 32.9, 34.6, 35.26, 35.33, 51.5, 128.7, 131.5, 133.0, 133.1, 135.2, 135.6, 136.2, 138.9, 139.69,

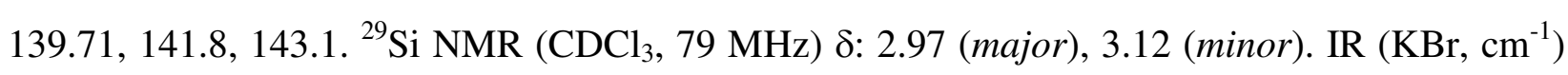
v: 2952, 2926, 1247, 1152, 1090, 1059, 1040, 885, 840, 721. MS (ESI): $\mathrm{m} / \mathrm{z}(\%) 713\left[(2 \mathrm{M}+\mathrm{H})^{+}\right.$, 10], 357 [(M + H $)^{+}$, 20], 239 (100). Anal. calcd for $\mathrm{C}_{21} \mathrm{H}_{28} \mathrm{OSSi}$ : C 70.73, H 7.91, S 8.99. Found: C 70.72, H 8.01, S 8.91.

$\left(S_{\mathrm{p}}, S_{\mathrm{s}}\right)$ *-4-Benzylsulfinyl[2.2]paracyclophane (9a). Obtained via the sulfenate with the general procedure described and using $\beta$-sulfinyl ester 5 (342 mg, $1.0 \mathrm{mmol})$ in dry THF (5 mL), $t$-BuOK $(1.12 \mathrm{~mL})$ and benzyl bromide $(132 \mu \mathrm{L}, 1.1 \mathrm{mmol})$ as the electrophile. Purification by chromatography ( $\left.\mathrm{EtOAc} / \mathrm{CH}_{2} \mathrm{Cl}_{2}, 1: 9\right)$ furnished exclusively the title sulfoxide $9 \mathrm{a}$ as a colourless solid (283 mg, $0.82 \mathrm{mmol}, 82 \%$ ). TLC (EtOAc/ $\left.\mathrm{CH}_{2} \mathrm{Cl}_{2}, 1: 9\right) R_{f}=0.60 . \mathrm{mp}: 122{ }^{\circ} \mathrm{C}$ $\left(\mathrm{CH}_{2} \mathrm{Cl}_{2} /\right.$ pentane). ${ }^{1} \mathrm{H} \mathrm{NMR}\left(\mathrm{CDCl}_{3}, 250 \mathrm{MHz}\right) \delta: 2.64(\mathrm{ddd}, 1 \mathrm{H}, J=5.4,10.0$ and 13.4), 2.95$3.21(\mathrm{~m}, 7 \mathrm{H}), 3.81$ and $3.94(\mathrm{AB}, 2 \mathrm{H}, J=12.5), 6.39-6.52(\mathrm{~m}, 4 \mathrm{H}), 6.62(\mathrm{dd}, 1 \mathrm{H}, J=1.9$ and 7.8$)$, 6.75-6.79 (m, 1H), $6.88(\mathrm{~d}, 1 \mathrm{H}, J=1.9), 6.94-6.99(\mathrm{~m}, 2 \mathrm{H}), 7.18-7.27(\mathrm{~m}, 3 \mathrm{H}) .{ }^{13} \mathrm{C}$ NMR $\left(\mathrm{CDCl}_{3}, 63 \mathrm{MHz}\right) \delta: 32.5,34.5,35.2,35.3,62.8,128.2,128.3,128.4,129.7,130.5,131.6,133.0$, 133.1 (2C), 134.7, 135.9, 137.0, 139.0, 139.6, 141.8, 142.3. IR $\left(\mathrm{KBr}, \mathrm{cm}^{-1}\right)$ v: 2922, 1073, 1064, 1036, 850, 761, 719, 696. MS (ESI): $m / z(\%) 1061\left[(3 \mathrm{M}+\mathrm{Na})^{+}, 13\right], 715\left[(2 \mathrm{M}+\mathrm{Na})^{+}, 85\right], 693$ $\left[(2 \mathrm{M}+\mathrm{H})^{+}, 44\right], 369\left[(\mathrm{M}+\mathrm{Na})^{+}, 45\right], 347\left[(\mathrm{M}+\mathrm{H})^{+}, 100\right]$. Anal. calcd for $\mathrm{C}_{23} \mathrm{H}_{22} \mathrm{OS}: \mathrm{C} 79.73$, H 6.40, S 9.25; Found: C 79.57, H 6.64, S 9.38.

Sulfoxide 9a was also formed as the sole product using a TBAF-induced fragmentation from 2-(trimethylsilyl)ethyl sulfoxide 6. A $1 \mathrm{M}$ solution of TBAF (previously dried on $4 \AA$ molecular sieves) in THF ( $840 \mu \mathrm{L}, 0.84 \mathrm{mmol}$ ) was added to a solution (1.6 mL THF) of 6 (143 $\mathrm{mg}, 0.4 \mathrm{mmol})$ and benzyl bromide $(57 \mu \mathrm{L}, 0.48 \mathrm{mmol})$. The reaction mixture was heated at 60 
${ }^{\circ} \mathrm{C}$ until completion according to TLC. The mixture was hydrolyzed with $\mathrm{H}_{2} \mathrm{O}(10 \mathrm{~mL})$ and THF was removed under vacuum. The aqueous layer was extracted with EtOAc (3 $510 \mathrm{~mL})$ then the organic layer was washed with saturated sodium chloride (2 $515 \mathrm{~mL})$, dried over $\mathrm{MgSO}_{4}$, filtered and evaporated to dryness. The crude product was purified by chromatography on silica gel (pentane/EtOAc, 4:1) to give 9a as a white solid (100 $\mathrm{mg}, 0,29 \mathrm{mmol}, 72 \%)$. Spectral data were similar to those reported above.

$\left(S_{\mathrm{p}}, S_{\mathrm{s}}\right) *-4-M e t h y l s u l f i n y l[2.2]$ paracyclophane $(9 \mathrm{~b})$. Obtained via the sulfenate with the general procedure described and using $\beta$-sulfinyl ester 5 (342 mg, $1.0 \mathrm{mmol})$ in dry THF (5 mL), $t$-BuOK $(1.1 \mathrm{~mL})$ and methyl iodide $(68 \mu \mathrm{L}, 1.1 \mathrm{mmol})$ as the electrophile. Purification by chromatography (EtOAc/ $\mathrm{CH}_{2} \mathrm{Cl}_{2}, 1: 1$ ) furnished exclusively the title sulfoxide $\mathbf{9 b}$ as a colourless solid (237 mg, $0.88 \mathrm{mmol}, 88 \%)$. TLC (EtOAc/ $\left.\mathrm{CH}_{2} \mathrm{Cl}_{2}, 3: 7\right) \quad R_{f}=0.37 . \mathrm{mp}: 153{ }^{\circ} \mathrm{C}$ $\left(\mathrm{CH}_{2} \mathrm{Cl}_{2} /\right.$ pentane). ${ }^{1} \mathrm{H} \mathrm{NMR}\left(\mathrm{CDCl}_{3}, 250 \mathrm{MHz}\right) \delta: 2.55$ (s, 3H), 2.86-2.96 (m, 1H), 3.04-3.35 (m, $7 \mathrm{H}), 6.47-6.56(\mathrm{~m}, 4 \mathrm{H}), 6.65(\mathrm{dd}, 1 \mathrm{H}, J=1.9$ and 7.7), 6.82-6.85 (m, 1H), 7.03 (d, 1H, $J=1.9)$. ${ }^{13} \mathrm{C} \mathrm{NMR}\left(\mathrm{CDCl}_{3}, 63 \mathrm{MHz}\right) \delta$ : 32.6, 34.6, 35.28, 35.34, 42.5, 127.6, 131.1, 133.09, 133.15, 133.2, 135.3, 135.5, 135.8, 138.9, 139.8, 142.3, 145.3. IR $\left(\mathrm{KBr}, \mathrm{cm}^{-1}\right)$ v: 2924, 1065, 1028, 959, 913, 848, 719, 696. MS (ESI): $m / z(\%) 563\left[(2 \mathrm{M}+\mathrm{Na})^{+}, 18\right], 541\left[(2 \mathrm{M}+\mathrm{H})^{+}, 7\right], 311(18), 293$ $\left[(\mathrm{M}+\mathrm{Na})^{+}, 56\right], 271\left(\mathrm{MH}^{+}, 100\right)$. Anal. calcd for $\mathrm{C}_{17} \mathrm{H}_{18} \mathrm{OS}: \mathrm{C} 75.51, \mathrm{H}$ 6.71, S 11.86; Found: C 75.17, H 6.69, S 11.69 .

$\left(S_{\mathrm{p}}, S_{\mathrm{s}}\right)^{*-4-E t h y l s u l f i n y l[2.2] p a r a c y c l o p h a n e ~(9 c) . ~ O b t a i n e d ~ v i a ~ t h e ~ s u l f e n a t e ~ w i t h ~ t h e ~}$ general procedure described and using $\beta$-sulfinyl ester 5 (342 mg, $1.0 \mathrm{mmol})$ in dry THF (5 mL), $t$-BuOK $(1.1 \mathrm{~mL})$ and ethyl iodide $(88 \mu \mathrm{L}, 1.1 \mathrm{mmol})$ as the electrophile. Purification by chromatography (EtOAc/ $\left.\mathrm{CH}_{2} \mathrm{Cl}_{2}, 1: 1\right)$ furnished exclusively the title sulfoxide 9c as a colourless solid (256 mg, $0.90 \mathrm{mmol}, 90 \%)$. TLC (EtOAc/ $\left.\mathrm{CH}_{2} \mathrm{Cl}_{2}, 1: 4\right) R_{f}=0.44 . \mathrm{mp}: 101{ }^{\circ} \mathrm{C}\left(\mathrm{CH}_{2} \mathrm{Cl}_{2}\right) .{ }^{1} \mathrm{H}$ NMR $\left(\mathrm{CDCl}_{3}, 250 \mathrm{MHz}\right) \delta: 1.09(\mathrm{t}, 3 \mathrm{H}, J=7.5), 2.58$ and 2.79 (AB part of $\mathrm{ABX}_{3}$ system, $1 \mathrm{H}$ each, $\left.J_{A B}=13.3, J_{A X}=J_{B X}=7.5\right), 2.85-2.95(\mathrm{~m}, 1 \mathrm{H}), 3.04-3.39(\mathrm{~m}, 7 \mathrm{H}), 6.46-6.57(\mathrm{~m}, 4 \mathrm{H}), 6.65$ $(\mathrm{dd}, 1 \mathrm{H}, J=1.8$ and 7.8$), 6.84-6.88(\mathrm{~m}, 1 \mathrm{H}), 6.98(\mathrm{~d}, 1 \mathrm{H}, J=1.8) .{ }^{13} \mathrm{C} \mathrm{NMR}\left(\mathrm{CDCl}_{3}, 63 \mathrm{MHz}\right) \delta$ : 6.1, 32.8, 34.6, 35.3, 35.4, 49.0, 128.7, 131.4, 133.1, 133.15, 133.2, 135.2, 135.7, 136.1, 139.0, 139.8, 141.8, 142.8. IR (KBr, cm $\left.{ }^{-1}\right)$ v: 2927, 1447, 1068, 1033, 1011, 969, 910, 846, 793, 719 $\mathrm{cm}^{-1}$. MS (ESI): $m / z(\%) 591\left[(2 \mathrm{M}+\mathrm{Na})^{+}, 18\right], 569\left[(2 \mathrm{M}+\mathrm{H})^{+}, 6\right], 325(12), 307\left[(\mathrm{M}+\mathrm{Na})^{+}\right.$, 26], $285\left[(\mathrm{M}+\mathrm{H})^{+}, 100\right]$. Anal. calcd for $\mathrm{C}_{18} \mathrm{H}_{20} \mathrm{OS}$ : C 76.01, H 7.09; Found: C 75.80, H 7.53.

\section{Oxidation of Sulfides 3}

Oxidation of benzyl sulfide 3a with $\mathrm{H}_{2} \mathrm{O}_{2}, N$-sulfonyloxaziridines 11a, 11b or MCPBA led to sulfoxides 9a and 10a in various diastereoisomeric ratios. Purification by chromatography on silica gel (EtOAc/ $\left.\mathrm{CH}_{2} \mathrm{Cl}_{2}, 1: 9\right)$ allowed perfect separation of $9 \mathbf{a}\left(R_{f}=0.6\right)$ and $\mathbf{9 b}\left(R_{f}=0.68\right)$. Spectral data of $\mathbf{9 a}$ were identical to those previously described. $\left(\boldsymbol{S}_{\mathbf{p}}, \boldsymbol{R}_{\mathbf{s}}\right)^{*-4}$ Benzylsulfinyl[2.2]paracyclophane (10a). TLC (EtOAc/ $\left.\mathrm{CH}_{2} \mathrm{Cl}_{2}, 1: 9\right) R_{f}=0.68 . \mathrm{mp}: 118{ }^{\circ} \mathrm{C} .{ }^{1} \mathrm{H}$ NMR $\left(\mathrm{CDCl}_{3}, 250 \mathrm{MHz}\right) \delta: 2.79-3.21(\mathrm{~m}, 6 \mathrm{H}), 3.34(\mathrm{ddd}, 1 \mathrm{H}, J=5.2,10.6$ and 12.5), 3.93-4.10 (m, 1H), 4.03 and $4.11(\mathrm{AB}, 2 \mathrm{H}, J=12.4), 6.42-6.61(\mathrm{~m}, 6 \mathrm{H}), 6.84(\mathrm{~d}, 1 \mathrm{H}, J=8.0), 6.95-6.99$ (m, 2H), 7.15-7.29 (m, 3H). ${ }^{13} \mathrm{C} \mathrm{NMR}\left(\mathrm{CDCl}_{3}, 63 \mathrm{MHz}\right) \delta: 33.2,35.0,35.3,36.0,62.0,128.1$, $128.5,130.3$, 130.4, 132.5, 132.6, 132.7, 132.8, 133.7, 136.5, 138.0, 138.8, 139.2, 140.4, 140.5, 
141.4. IR (KBr, $\left.\mathrm{cm}^{-1}\right)$ v: $2921,1059,1047,891,850,770,718,702$. MS (ESI): $\mathrm{m} / \mathrm{z}(\%) 347$ [(M $\left.+\mathrm{H})^{+}, 58\right], 329$ (21), 175 (100), 239 (100), 91 (15). HRMS (ESI) Calcd for $\mathrm{C}_{23} \mathrm{H}_{23} \mathrm{OS}: 347.1470$, Found: 347.1456. Anal. calcd for $\mathrm{C}_{23} \mathrm{H}_{22} \mathrm{OS}$ : C 79.73, H 6.40; Found: C 79.50, H 6.67.

Oxidation of methyl sulfide $\mathbf{3 b}$ with oxaziridine $11 \mathbf{b}$ on a $0.5 \mathrm{mmol}$ scale, as described in the General Procedures above, led to diastereomeric sulfoxides 9b and 10b in a 9:91 ratio. Purification by chromatography on silica gel (EtOAc/ $\left.\mathrm{CH}_{2} \mathrm{Cl}_{2}, 3: 7\right)$ allowed perfect separation of 9b $\left(R_{f}=0.37\right)$ and 10b $\left(R_{f}=0.29\right)$ with an overall yield of $90 \%(122 \mathrm{mg}, 0.45 \mathrm{mmol})$. Spectral data of $\mathbf{9 b}$ are identical to those previously described in the Supporting Information. $\left(\boldsymbol{S}_{\mathrm{p}}, \boldsymbol{R}_{\mathrm{s}}\right) *-\mathbf{4}$ Methylsulfinyl[2.2]paracyclophane (10b). TLC (EtOAc/CH $\left.\mathrm{Cl}_{2}, 3: 7\right) R_{f}=0.29 . \mathrm{mp}: 142{ }^{\circ} \mathrm{C} .{ }^{1} \mathrm{H}$ NMR $\left(\mathrm{CDCl}_{3}, 250 \mathrm{MHz}\right) \delta: 2.74(\mathrm{~s}, 3 \mathrm{H}), 2.93-3.23(\mathrm{~m}, 6 \mathrm{H}), 3.33$ (ddd, $1 \mathrm{H}, J=5.9,10.0$ and 12.4), 4.16 (ddd, $1 \mathrm{H}, J=2.0,10.0$ and 12.4), $6.43(\mathrm{~d}, 1 \mathrm{H}, J=8.1), 6.53-6.63(\mathrm{~m}, 5 \mathrm{H}), 6.78(\mathrm{~d}$, $1 \mathrm{H}, J=8.1) .{ }^{13} \mathrm{C} \mathrm{NMR}\left(\mathrm{CDCl}_{3}, 63 \mathrm{MHz}\right) \delta: 33.0,35.0,35.3,35.8,41.0,130.8,132.6,132.7$, $132.9,133.1,136.5,137.9,139.3,140.1,140.6,141.1,141.4 . \mathrm{IR}\left(\mathrm{KBr}, \mathrm{cm}^{-1}\right)$ v: 2926, 1059, 1023, 954, 937, 909, 899, 845, 795, 720. MS (ESI): $m / z(\%) 271\left[(\mathrm{M}+\mathrm{H})^{+}, 56\right], 256(16), 167$ (100), 105 (24). HRMS (ESI) Calcd for $\mathrm{C}_{17} \mathrm{H}_{19}$ OS: 271.1157, Found: 271.1164. Anal. calcd for $\mathrm{C}_{17} \mathrm{H}_{18} \mathrm{OS}$ : C 75.51, H 6.71, S 11.86; Found: C 75.36, H 6.90, S 12.16.

Oxidation of ethyl sulfide $3 \mathbf{c}$ with oxaziridine $11 \mathbf{b}$ on a $0.5 \mathrm{mmol}$ scale, as described in the General Procedures above, led to diastereomeric sulfoxides 9c and 10c in a 19:81 ratio. Purification by chromatography on silica gel $\left(\mathrm{EtOAc} / \mathrm{CH}_{2} \mathrm{Cl}_{2}, 1: 4\right)$ did not allow separation of both isomers. The indivisible products were isolated as a white solid $(117 \mathrm{mg}, 0.41 \mathrm{mmol}, 82 \%)$ displaying the following data. TLC (EtOAc/ $\left.\mathrm{CH}_{2} \mathrm{Cl}_{2}, 1: 4\right) R_{f}=0.44$. mp: $54{ }^{\circ} \mathrm{C} .{ }^{1} \mathrm{H} \mathrm{NMR}\left(\mathrm{CDCl}_{3}\right.$, $250 \mathrm{MHz}) \delta: 1.08(\mathrm{t}, 0.6 \mathrm{H}, J=7.4$, minor $), 1.10(\mathrm{t}, 2.4 \mathrm{H}, J=7.5$, major $), 2.49-2.63(\mathrm{~m}, 0.2 \mathrm{H}$, minor), 2.71-3.40 (m, 9H, minor), 4.18 (ddd, 0.8H, $J=2.2,10.4$ and 12.5, major), 6.44-6.66 (m, $5.8 \mathrm{H}$, major + minor $), 6.83-6.88(\mathrm{~m}, 1 \mathrm{H}$, major + minor $), 6.97(\mathrm{~d}, 0.2 \mathrm{H}, J=2.0$, minor $) .{ }^{13} \mathrm{C}$ $\mathrm{NMR}\left(\mathrm{CDCl}_{3}, 63 \mathrm{MHz}\right) \delta$ (major): 7.5, 33.2, 35.0, 35.3, 35.9, 48.8, 132.1, 132.58, 132.64, 132.7, $133.4,136.3,137.9,139.2,139.4,140.2,140.7,141.1 .{ }^{13} \mathrm{C} \mathrm{NMR}\left(\mathrm{CDCl}_{3}, 63 \mathrm{MHz}\right) \delta$ (minor): 5.9, 32.6, 34.5, 35.1, 35.2, 48.8, 128.6, 131.2, 132.9, 133.0, 133.1, 135.1, 135.6, 136.0, 138.8, 139.6, 141.7, 142.6. MS (ESI): $\mathrm{m} / z(\%) 285$ [(M + H) $\left.{ }^{+}, 69\right], 256$ (16), 208 (16), 181 (100), 153 (55), 135 (18), 105 (18). HRMS (ESI) Calcd for $\mathrm{C}_{18} \mathrm{H}_{21} \mathrm{OS}$ : 285.1313, Found: 285.1311. 


\section{Theoretical Calculations}

A rapid conformation analysis of the sulfenate species using a semi-empirical method (PM3-Spartan program $)^{7}$ led to the identication of two stable conformers in which the S-O bond lies in the plane of the aromatic ring. Intermediate II has the oxygen pointing towards the ethylene bridge, while in intermediate $\mathbf{I}$ it points to the opposite direction. To obtain reliable energy values, geometry optimizations for both intermediates have been investigated (Spartan software) using HF and hybrid DFT methods, as reported in Table 1.

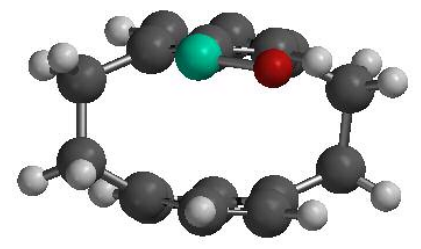

Sulfenate conformer I

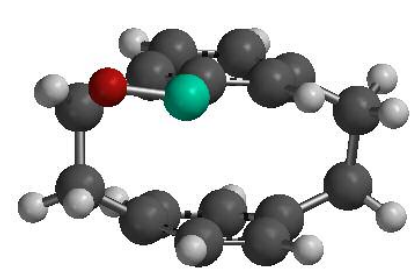

Sulfenate conformer II

\begin{tabular}{|l|c|c|c|c|}
\hline & HF-6-31+G* & HF-6-311+G** & B3LYP/6-31+G* & B3LYP/6-311+G** \\
\hline Intermediate I & 0 & 0 & 0 & 0 \\
\hline Intermediate II & 2.23 & 2.13 & 1.78 & 1.77 \\
\hline
\end{tabular}

Table 1: Relative energies (Kcal/mol) of sulfenates I and II.

The results indicate that sulfenate $\mathbf{I}$ is the thermodynamic intermediate. The energy differences between I and II are almost identical whatever the Hartree-Fock method used, while a smaller difference is observed by using DFT methods. In view of these results which are otherwise quite close, and anticipating the calculation time needed to model the transition state structures, we decided to achieve the next calculations by using the HF/6-31+G* level of theory. Gaussian software ${ }^{8}$ was used for the next calculations, all the stationary points found being characterized by the correct number and nature of negative eigenvalues of the Hessian matrix. First of all, the transition structure (TS) TST0 corresponding to the cross-exchange of intermediate I to intermediate II was determined. The frequency analysis (only one imaginary frequency corresponding to the reaction coordinate) confirmed the existence of a TS. The energy of this TS is $11.50 \mathrm{Kcal} / \mathrm{mol}$ (E0) higher than the most stable sulfenate I. The approach of the

\footnotetext{
${ }^{7}$ Wavefunction, Spartan '04.
}

${ }^{8}$ Gaussian 03, Revision C.02, Frisch, M. J.; Trucks, G. W.; Schlegel, H. B.; Scuseria, G. E.; Robb, M. A.; Cheeseman, J. R.; Montgomery, Jr., J. A.; Vreven, T.; Kudin, K. N.; Burant, J. C.; Millam, J. M.; Iyengar, S. S.; Tomasi, J.; Barone, V.; Mennucci, B.; Cossi, M.; Scalmani, G.; Rega, N.; Petersson, G. A.; Nakatsuji, H.; Hada, M.; Ehara, M.; Toyota, K.; Fukuda, R.; Hasegawa, J.; Ishida, M.; Nakajima, T.; Honda, Y.; Kitao, O.; Nakai, H.; Klene, M.; Li, X.; Knox, J. E.; Hratchian, H. P.; Cross, J. B.; Bakken, V.; Adamo, C.; Jaramillo, J.; Gomperts, R.; Stratmann, R. E.; Yazyev, O.; Austin, A. J.; Cammi, R.; Pomelli, C.; Ochterski, J. W.; Ayala, P. Y.; Morokuma, K.; Voth, G. A.; Salvador, P.; Dannenberg, J. J.; Zakrzewski, V. G.; Dapprich, S.; Daniels, A. D.; Strain, M. C.; Farkas, O.; Malick, D. K.; Rabuck, A. D.; Raghavachari, K.; Foresman, J. B.; Ortiz, J. V.; Cui, Q.; Baboul, A. G.; Clifford, S.; Cioslowski, J.; Stefanov, B. B.; Liu, G.; Liashenko, A.; Piskorz, P.; Komaromi, I.; Martin, R. L.; Fox, D. J.; Keith, T.; Al-Laham, M. A.; Peng, C. Y.; Nanayakkara, A.; Challacombe, M.; Gill, P. M. W.; Johnson, B.; Chen, W.; Wong, M. W.; Gonzalez, C.; and Pople, J. A.; Gaussian, Inc., Wallingford CT, 2004. 
electrophile according to a $\mathrm{S}_{\mathrm{N}} 2$ type reaction was investigated on $\mathbf{I}$ and $\mathbf{I I}$, leading respectively to the TST1 and TST2 transition structures. Chloromethane was selected as a model electrophile, instead of iodomethane used experimentally, to decrease the calculation times. For the energy of these TS we anticipate the importance of the entropic factor associated with a $\mathrm{S}_{\mathrm{N}} 2$ type reaction. For this reason we calculated the electronic energy $\left(\mathrm{E}_{0}\right)$, but also the Gibbs free energy $(\mathrm{G})$. All the results are summarized in Table 2 and Scheme 1 (relative Gibbs free energy along the reaction coordinate). From these results a negative entropic contribution is clearly observed for TST1 and TST2 while the unimolecular transition state TST0 is not affected.

\begin{tabular}{|c|c|c|c|c|c|c|c|}
\hline & I & II & TST0 & TST1 & TST2 & PI & PII \\
\hline$\Delta \mathrm{E}$ & 0 & 2.23 & 11.50 & 10.93 & 13.34 & -14.29 & -12.74 \\
\hline$\Delta \mathrm{G}$ & 0 & 2.13 & 11.46 & 21.28 & 23.74 & -10.42 & -8.50 \\
\hline
\end{tabular}

Table 2: Relative energies (Kcal/mol) for intermediates I and II, TST1, TST2, TST3 transition structures, and products $\mathbf{P 1}$ and $\mathbf{P 2}$, based on calculations using the HF/6-31+G* level of theory.

The reaction pathways corresponding to the alkylation of sulfenates I and II with chloromethane are depicted on Scheme 1 ( $\Delta \mathrm{G}$ along the reaction coordinate). This representation corresponds to a Curtin-Hammett system where the selectivity arises from the energy differences between TST1 and TST2 to produce the major product PI. In view of the energy difference between TST1 and TST2 the theoretical ratio PI/PII should be 98:2. These theoretical results are in good agreement with the experiments (a single diastereoisomer was detected).

It should be noted that the energy difference between TST1 and TST2 is almost coincident with that of intermediates $\mathbf{I}$ and $\mathbf{I I}$, indicating that the origin of the stereoselectivity probably lies in the destabilizing interactions presents in intermediate II between the sulfenate oxygen atom and the bridging methylene group, which are translated to the TS, due to its early nature. 


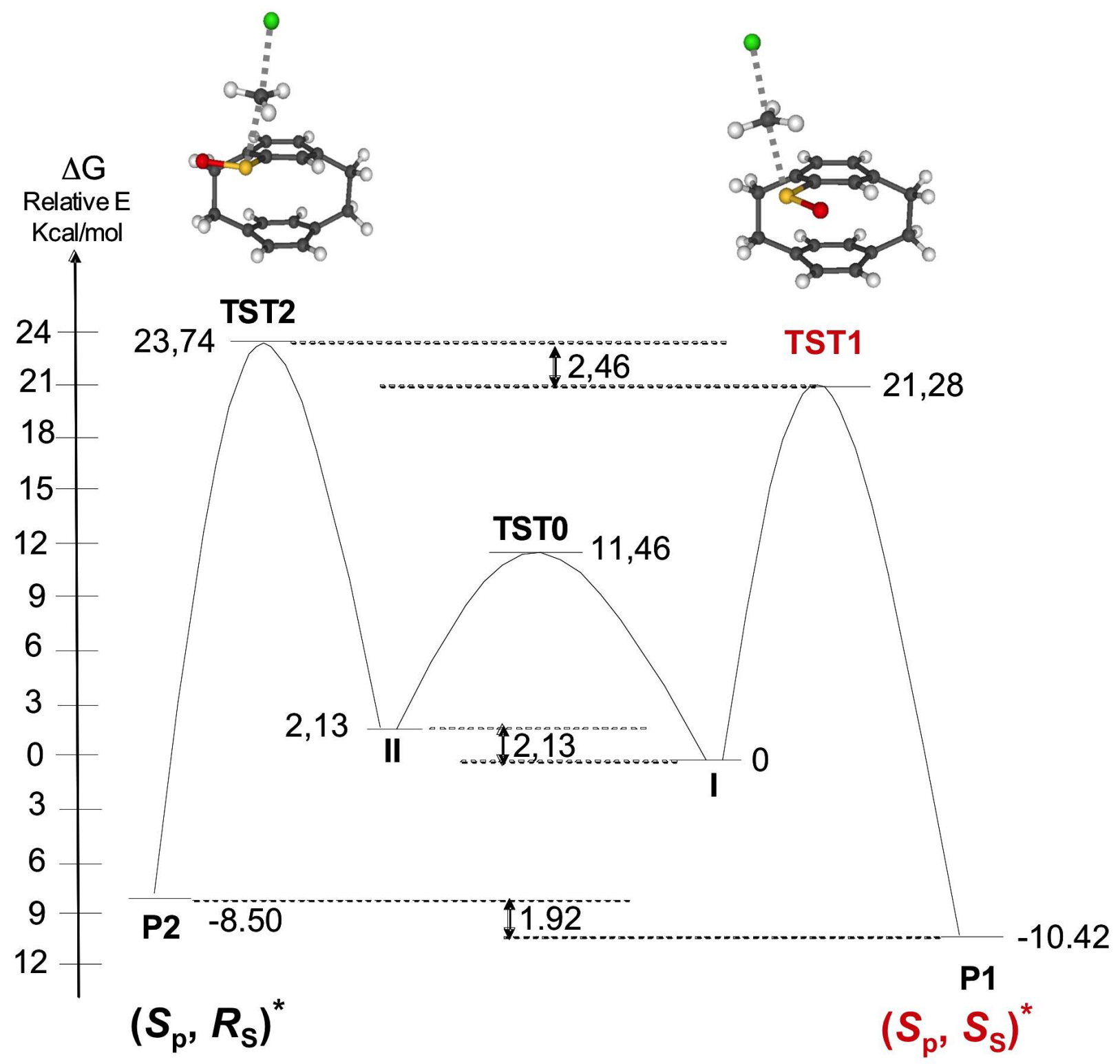

Scheme 1: Calculated $\left(\mathrm{HF} / 6-31+\mathrm{G}^{*}\right)$ reaction pathway. 


\section{Crystal Structure Determination of 9a}

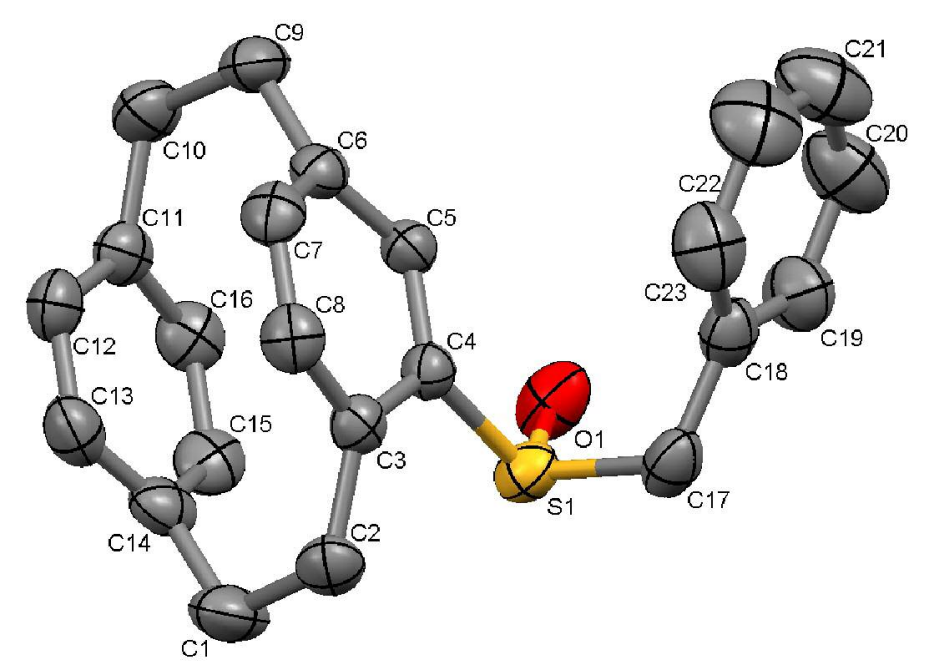

Single crystals of sulfoxide 9a suitable for X-ray crystallographic analysis were obtained by slow diffusion of pentane in $\mathrm{CH}_{2} \mathrm{Cl}_{2}$ solution. X-ray diffraction experiments for monocrystal of $9 \mathbf{a}$ were performed at $293 \mathrm{~K}$ with graphite-monochromatized Mo $\mathrm{K}_{\alpha}$ radiation on an BrukerNonius Kappa CCD area detector diffractometer. Formula $\mathrm{C}_{23} \mathrm{H}_{22} \mathrm{O}_{\mathrm{S}}$, formula weight 346.47, crystal system orthorhombic, space group Pbca, $a=11.0748(2) \AA, b=13.0716(3) \AA, c=$ 25.1947(5) $\AA, \alpha=90^{\circ}, \beta=90^{\circ}, \gamma=90^{\circ}, V=3647.32(13) \AA^{3}, Z=8$, calculated density = 1.262 $\mathrm{g} / \mathrm{cm}^{3}, \mu=0.185 \mathrm{~mm}^{-1}, \mathrm{R}_{\mathrm{int}}=0.043, \mathrm{R}\left[F^{2}>2 \sigma\left(F^{2}\right)\right]=0.0437, \mathrm{wR}\left(F^{2}\right)=0.1379$. Selected bond lengths $(\AA)$, angles (deg) and dihedral angles (deg): S1-O1 1.4878(14), S1-C4 1.7891(16), S1C17 1.842(2), O1-S1-C4 106.59(8), O1-S1-C17 107.16(9), O1-S1-C4-C5 -8.63(15). Program(s) used to solve structure: SHELXS-97. Program(s) used to refine structure: SHELXL-97. Software used to prepare material for publication: SHELXTL-97. Crystallographic data for compound 9a have been deposited at the Cambridge Crystallographic Data Centre, CCDC No 665854. Copies of this information may be obtained free of charge from The Director, CCDC, 12 Union Road, Cambridge, CB2 1EZ, UK (+44-1223-336408; E-mail: deposit@ccdc.cam.ac.uk or http://www.ccdc.cam.ac.uk). 


\section{Crystal Structure Determination of 9b}

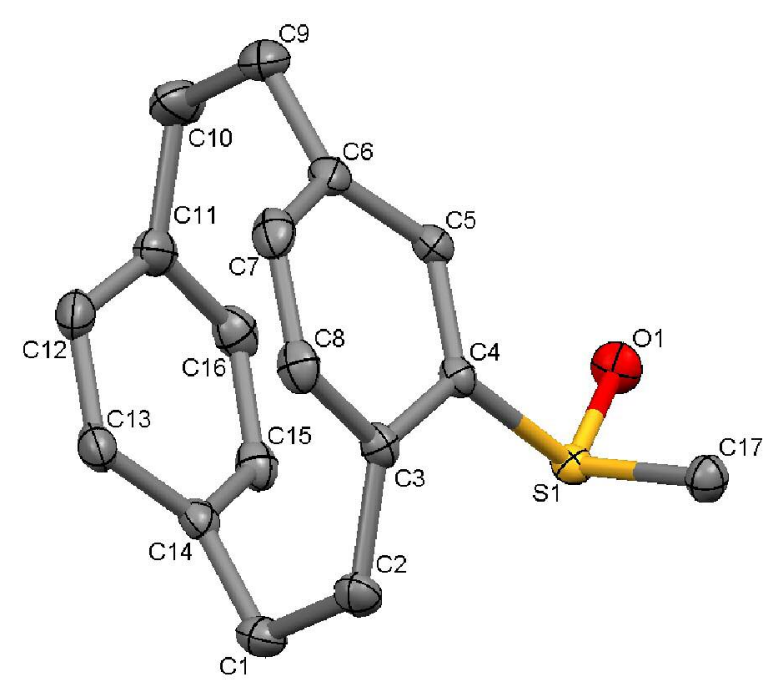

Single crystals of sulfoxide $\mathbf{9 b}$ suitable for X-ray crystallographic analysis were obtained by slow diffusion of pentane in $\mathrm{CH}_{2} \mathrm{Cl}_{2}$ solution. X-ray diffraction experiments for monocrystal of $9 \mathrm{a}$ were performed at $150 \mathrm{~K}$ with graphite-monochromatized Mo $\mathrm{K}_{\alpha}$ radiation on an BrukerNonius Kappa CCD area detector diffractometer. Formula $\mathrm{C}_{17} \mathrm{H}_{18} \mathrm{O}_{S}$, formula weight 270.37, crystal system monoclinic, space group P2(1)/c, $a=13.8811(9) \AA, b=9.2532(6) \AA ⿻$ 10.6560(7) $\AA, \alpha=90^{\circ}, \beta=93.048(4)^{\circ}, \gamma=90^{\circ}, V=1366.77(15) \AA^{3}, Z=4$, calculated density = $1.314 \mathrm{~g} / \mathrm{cm}^{3}, \mu=0.226 \mathrm{~mm}^{-1}, \mathrm{R}_{\mathrm{int}}=0.028, \mathrm{R}\left[F^{2}>2 \sigma\left(F^{2}\right)\right]=0.0357, \mathrm{wR}\left(F^{2}\right)=0.1186$. Selected bond lengths $(\AA)$, angles (deg) and dihedral angles (deg): S1-O1 1.4989(7), S1-C4 1.7928(8), S1-C17 1.7874(9), O1-S1-C4 106.52(4), O1-S1-C17 106.03(4), C4-C5-S1-O1 0.42(7). Program(s) used to solve structure: SHELXS-97. Program(s) used to refine structure: SHELXL97. Software used to prepare material for publication: SHELXTL-97. Crystallographic data for compound 9b have been deposited at the Cambridge Crystallographic Data Centre, CCDC No 666354. Copies of this information may be obtained free of charge from The Director, CCDC, 12 Union Road, Cambridge, CB2 1EZ, UK (+44-1223-336408; E-mail: deposit@ccdc.cam.ac.uk or http://www.ccdc.cam.ac.uk). 


\section{Crystal Structure Determination of 9c}

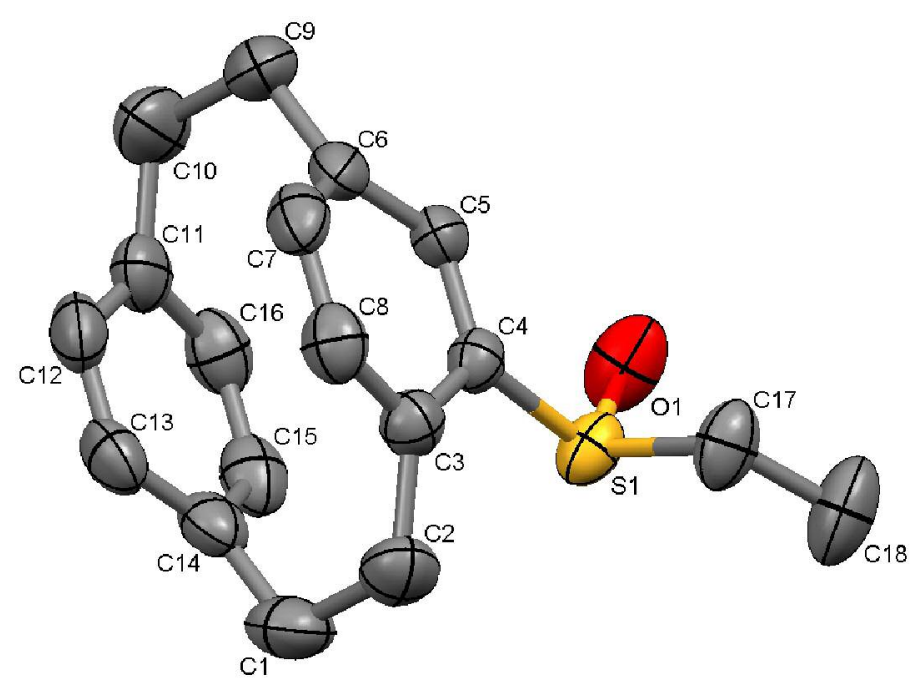

Single crystals of sulfoxide 9c suitable for X-ray crystallographic analysis were obtained by slow diffusion of pentane in $\mathrm{CH}_{2} \mathrm{Cl}_{2}$ solution. X-ray diffraction experiments for monocrystal of 9a were performed at $298 \mathrm{~K}$ with graphite-monochromatized Mo $\mathrm{K}_{\alpha}$ radiation on an BrukerNonius Kappa CCD area detector diffractometer. Formula $\mathrm{C}_{18} \mathrm{H}_{20} \mathrm{O}_{\mathrm{S}}$, formula weight 284.40, crystal system monoclinic, space group $\mathrm{P} 2(1) / \mathrm{c}, a=15.5982(5) \AA, b=8.7362(3) \AA, c=$ 11.1429(4) $\AA, \alpha=90^{\circ}, \beta=91.457(2)^{\circ}, \gamma=90^{\circ}, \mathrm{V}=1517.94(9) \AA^{3}, \mathrm{Z}=4$, calculated density $=$ $1.244 \mathrm{~g} / \mathrm{cm}^{3}, \mu=0.207 \mathrm{~mm}^{-1}, \mathrm{R}_{\text {int }}=0.033, \mathrm{R}\left[F^{2}>2 \sigma\left(F^{2}\right)\right]=0.0478, w \mathrm{w}\left(F^{2}\right)=0.1610$. Selected bond lengths (A), angles (deg) and dihedral angles (deg): S1-O1 1.4811(12), S1-C4 1.7956(12), S1-C17 1.8047(15), O1-S1-C4 107.79(6), O1-S1-C17 106.01(9), O1-S1-C4-C5 1.80(13). Program(s) used to solve structure: SHELXS-97. Program(s) used to refine structure: SHELXL97. Software used to prepare material for publication: SHELXTL-97. Crystallographic data for compound 9c have been deposited at the Cambridge Crystallographic Data Centre, CCDC No 665855. Copies of this information may be obtained free of charge from The Director, CCDC, 12 Union Road, Cambridge, CB2 1EZ, UK (+44-1223-336408; E-mail: deposit@ccdc.cam.ac.uk or http://www.ccdc.cam.ac.uk). 
${ }^{1} \mathrm{H}$ NMR $\left(\mathrm{CDCl}_{3}, 250 \mathrm{MHz}\right)$ of 12<smiles>COCCCSCCCC(=O)OC</smiles>

12

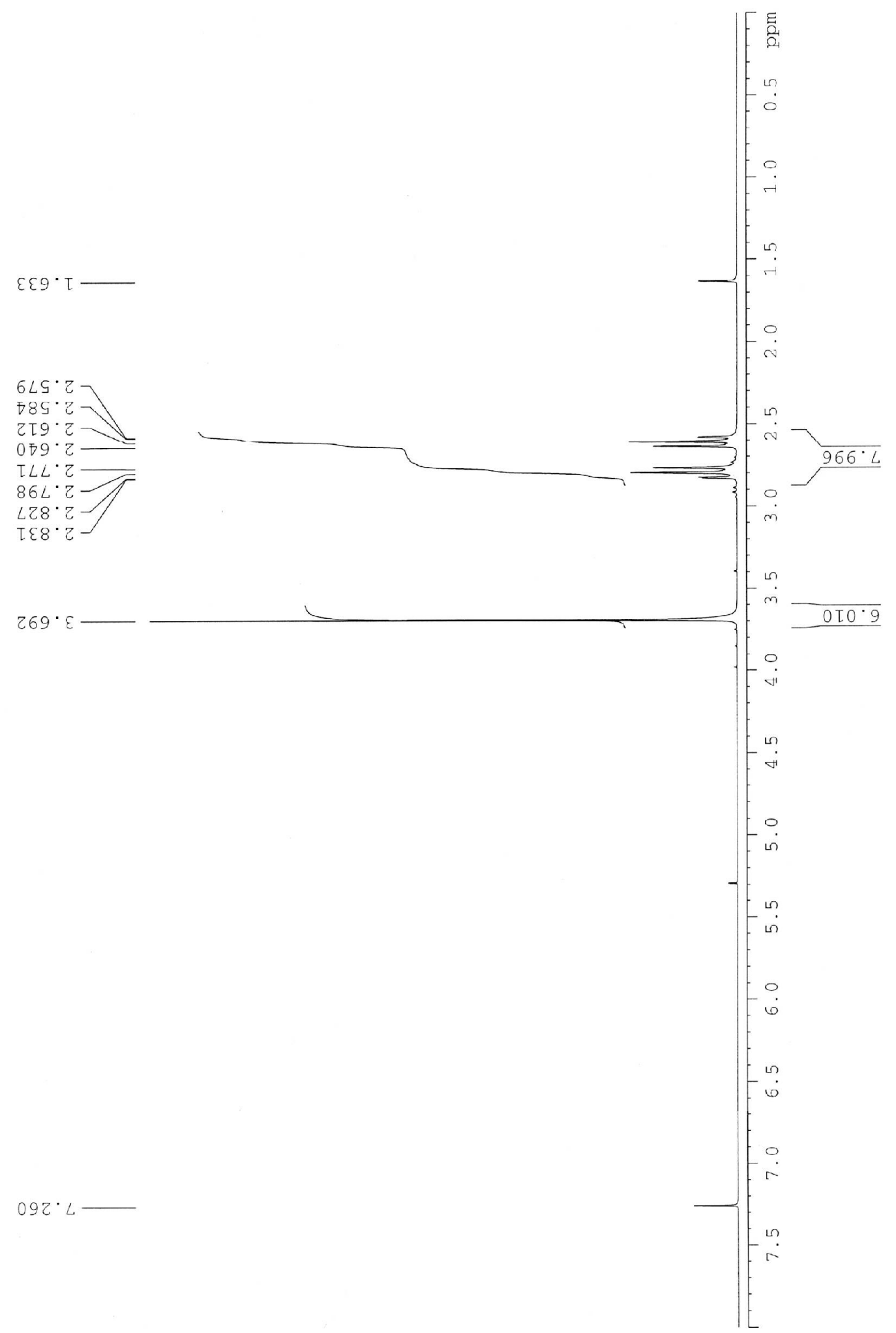


S17

${ }^{13} \mathrm{C} \mathrm{NMR}\left(\mathrm{CDCl}_{3}, 63 \mathrm{MHz}\right)$ of 12

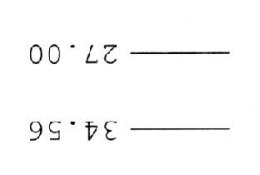

ร8. โร

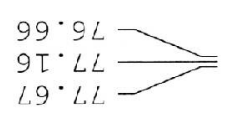

$\angle Z \cdot Z L T$
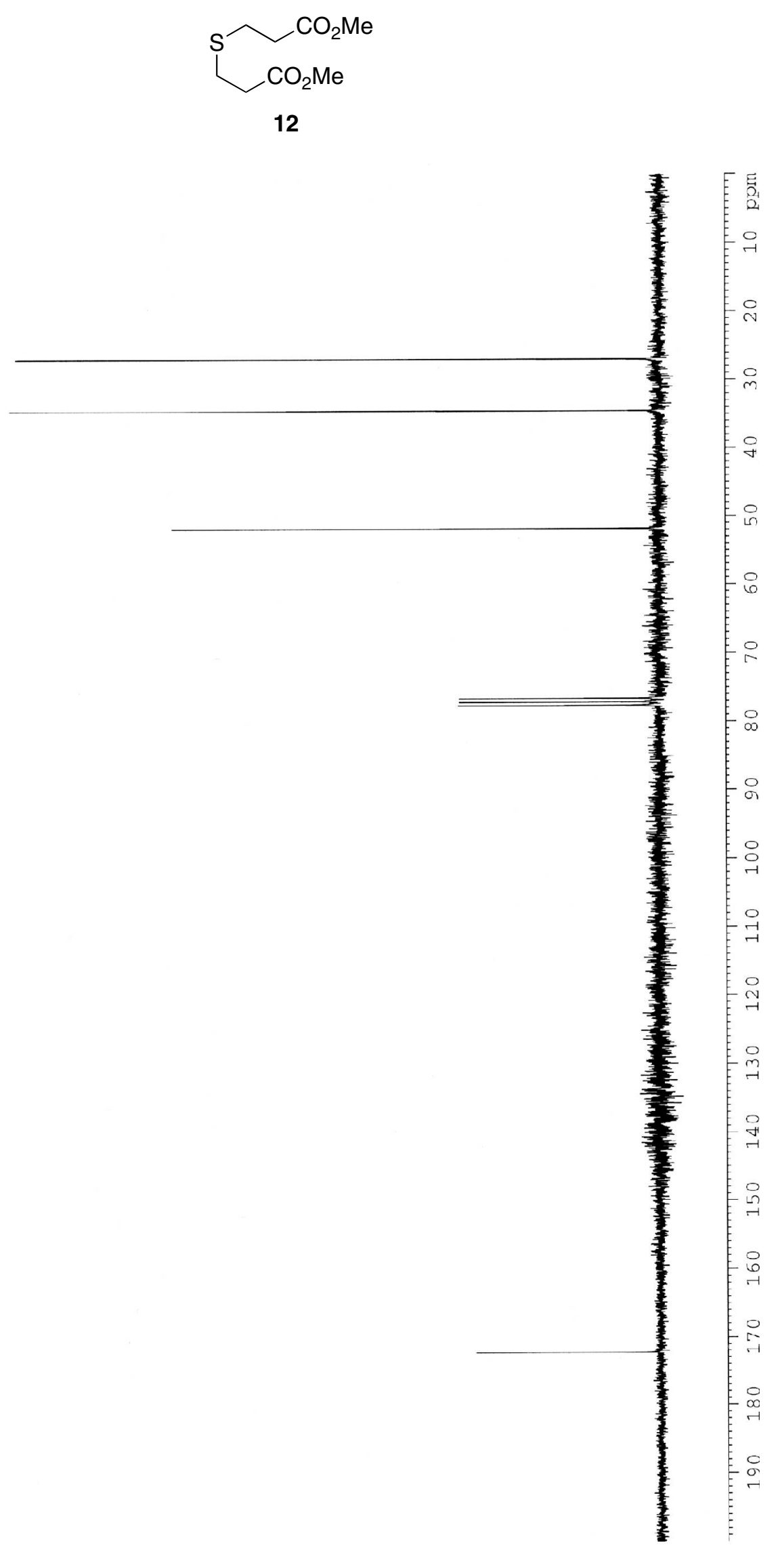
${ }^{1} \mathrm{H}$ NMR $\left(\mathrm{CDCl}_{3}, 250 \mathrm{MHz}\right)$ of 7
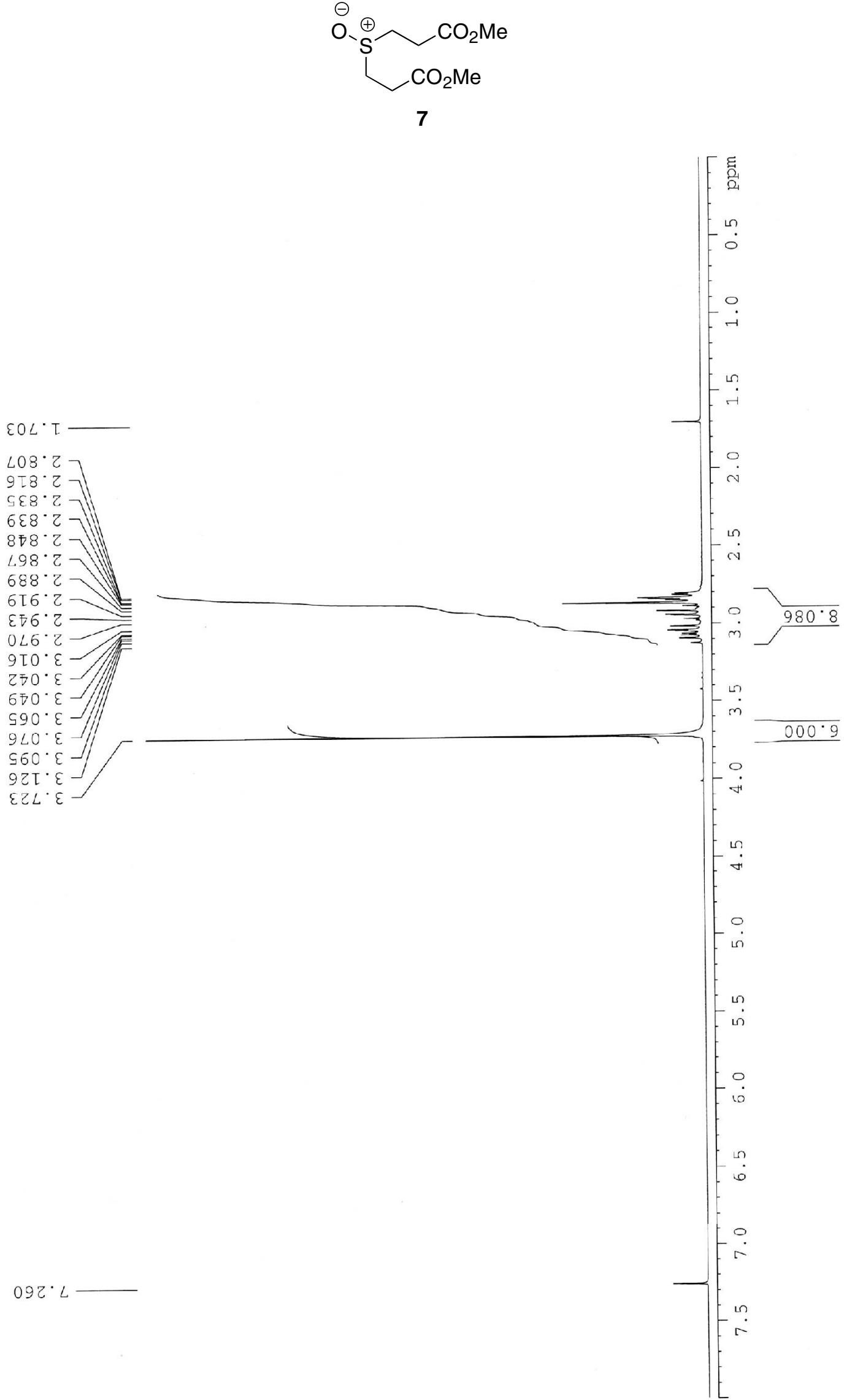
S19

${ }^{13} \mathrm{C} \mathrm{NMR}\left(\mathrm{CDCl}_{3}, 63 \mathrm{MHz}\right)$ of 7

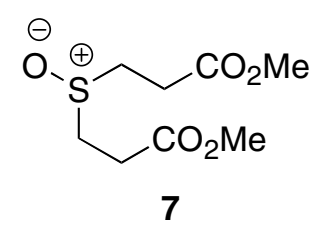

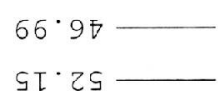

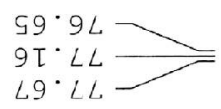

$\bar{\tau} \Im^{\cdot} \mathrm{TLI}$

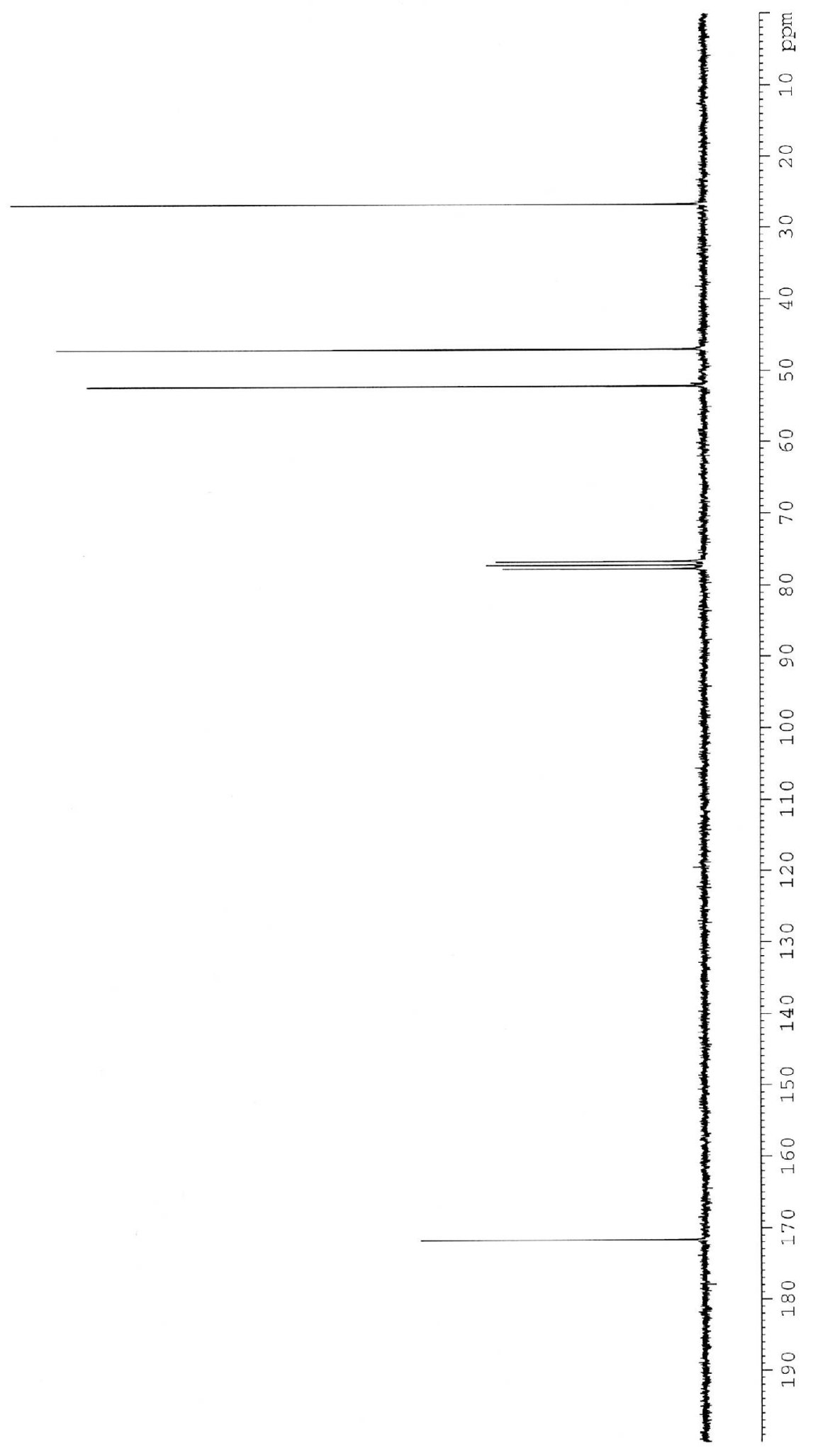


S20

${ }^{1} \mathrm{H}$ NMR $\left(\mathrm{CDCl}_{3}, 250 \mathrm{MHz}\right)$ of 4<smiles>CC(=O)CCSc1ccccc1CCc1ccc2c(c1)CCC2</smiles>

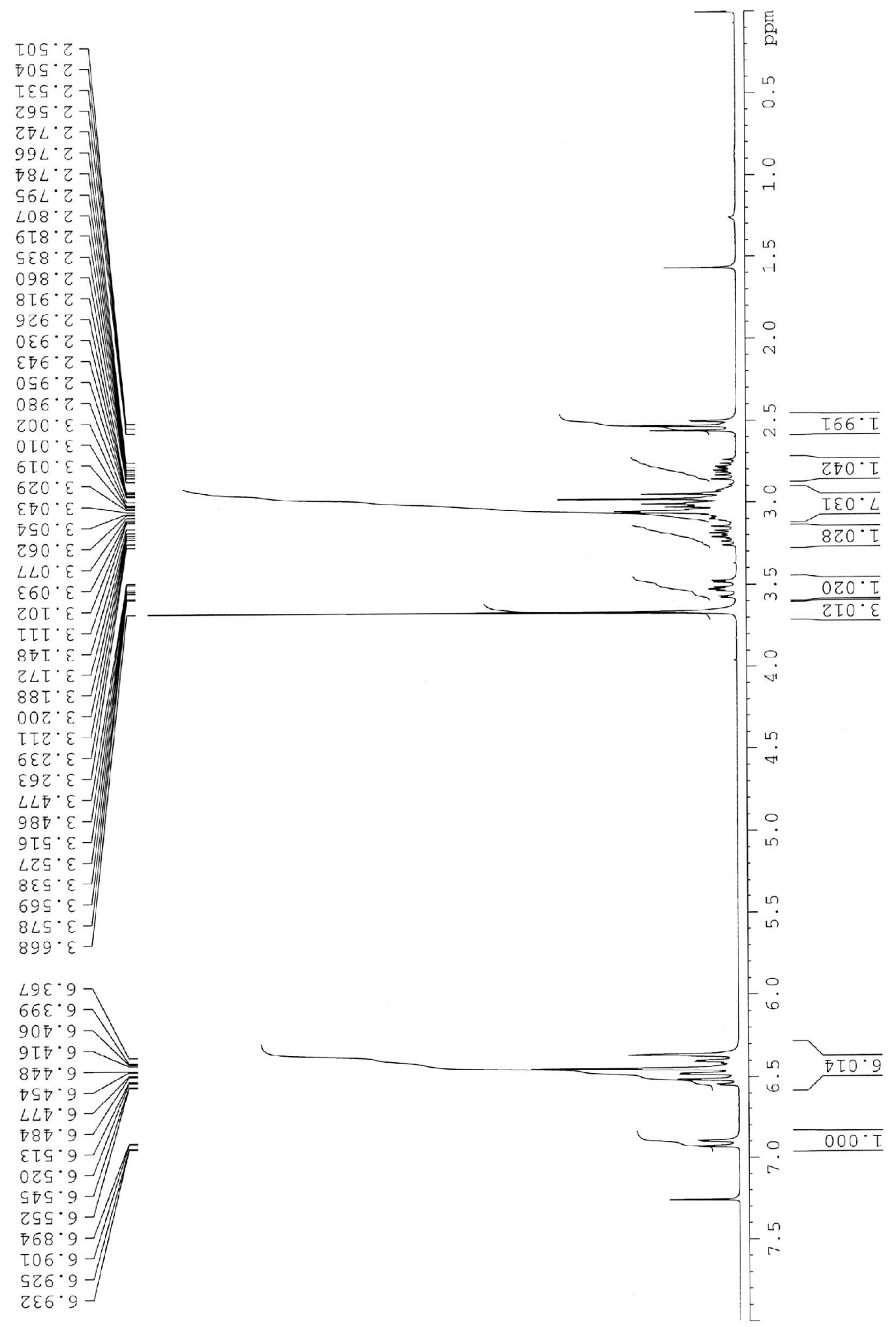




$$
1
$$


S22

${ }^{1} \mathrm{H} \mathrm{NMR}\left(\mathrm{CDCl}_{3}, 250 \mathrm{MHz}\right)$ of 2<smiles>Sc1cc2c(cc1CCc1ccccc1)CC2</smiles>

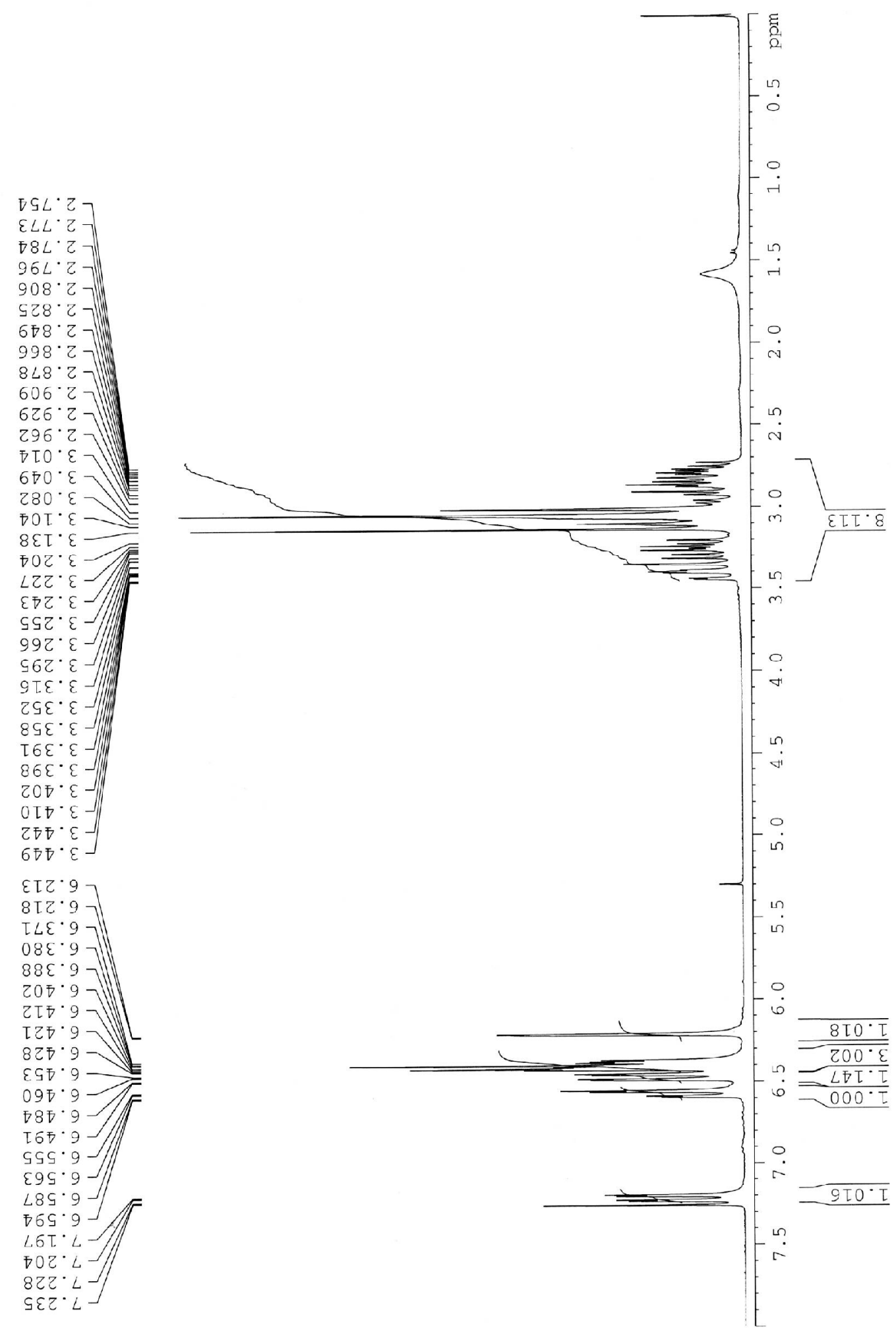




$$
4
$$


S24

${ }^{1} \mathrm{H}$ NMR $\left(\mathrm{CDCl}_{3}, 400 \mathrm{MHz}\right)$ of $3 \mathrm{a}$<smiles>Brc1ccccc1C1=CC2=CC=CC(=C1)CC2</smiles>

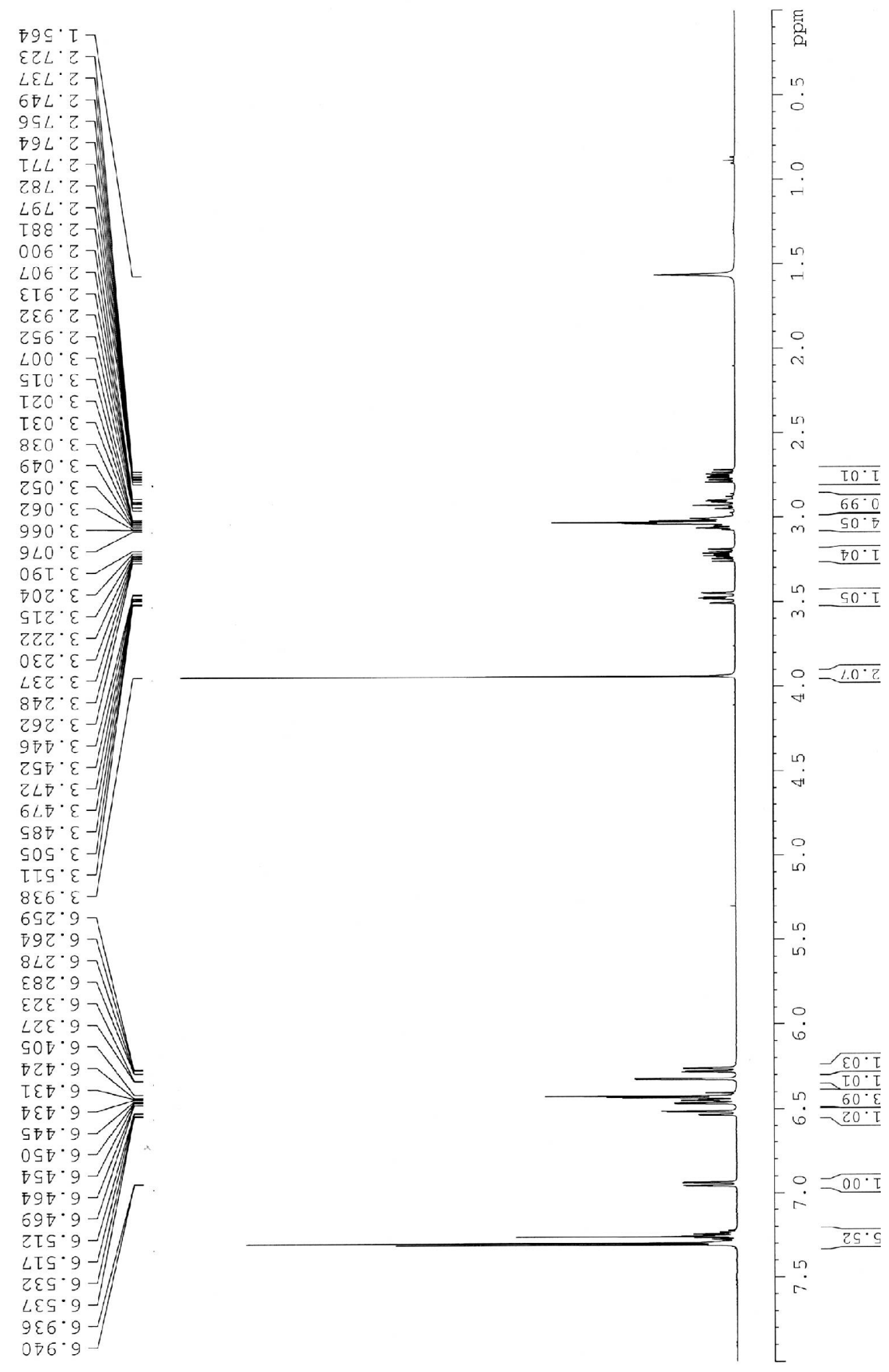




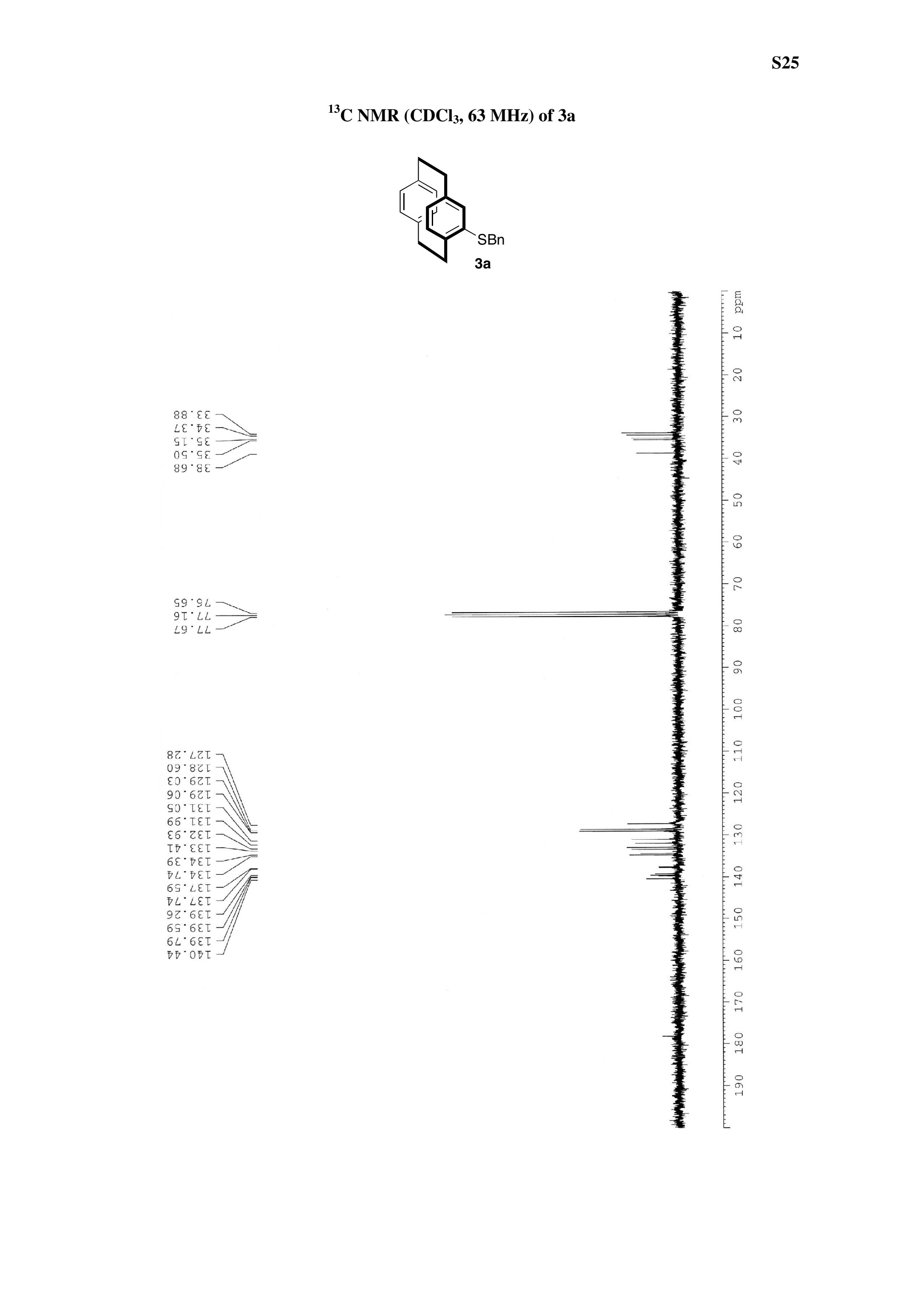


S26

${ }^{1} \mathrm{H}$ NMR $\left(\mathrm{CDCl}_{3}, 63 \mathrm{MHz}\right)$ of $3 \mathrm{~b}$<smiles>Cc1cc2ccc1CCC2</smiles>

3

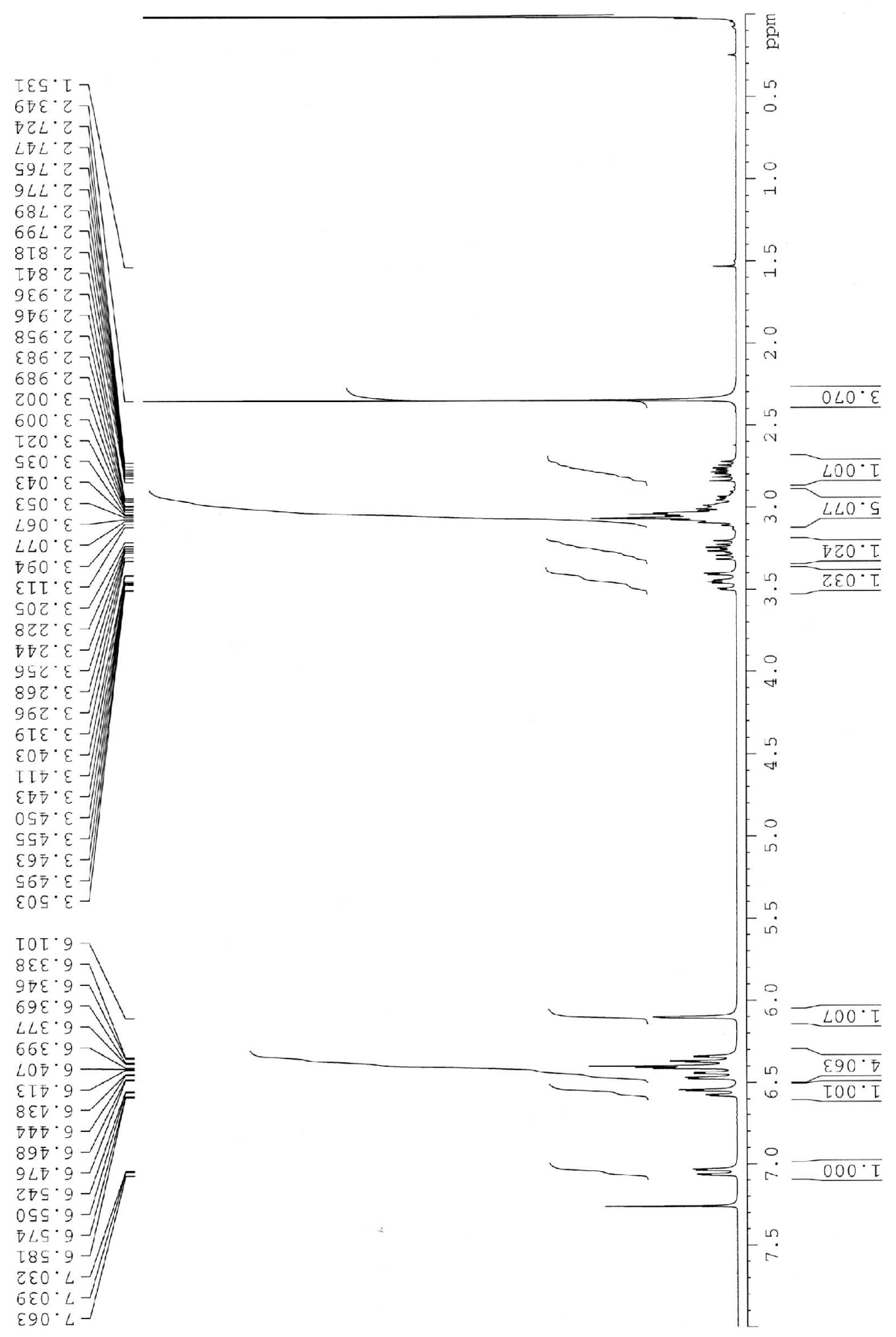


S27

${ }^{13} \mathrm{C} \mathrm{NMR}\left(\mathrm{CDCl}_{3}, 63 \mathrm{MHz}\right)$ of $3 \mathrm{~b}$
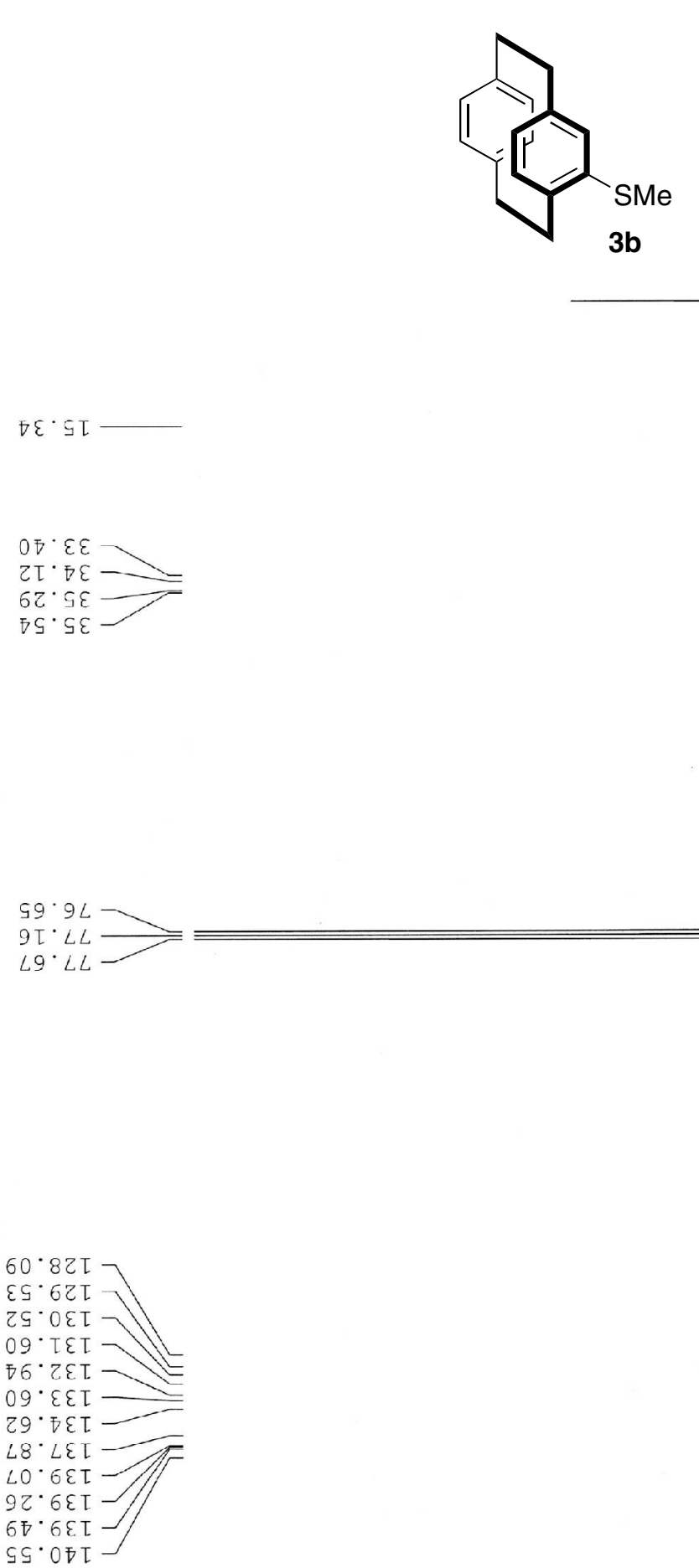

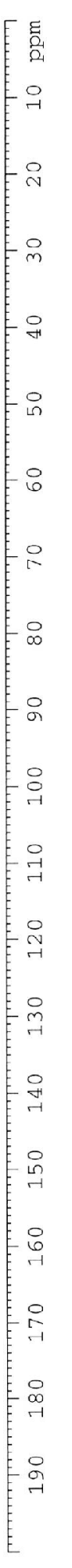


${ }^{1} \mathrm{H}$ NMR $\left(\mathrm{CDCl}_{3}, 250 \mathrm{MHz}\right)$ of $3 \mathrm{c}$
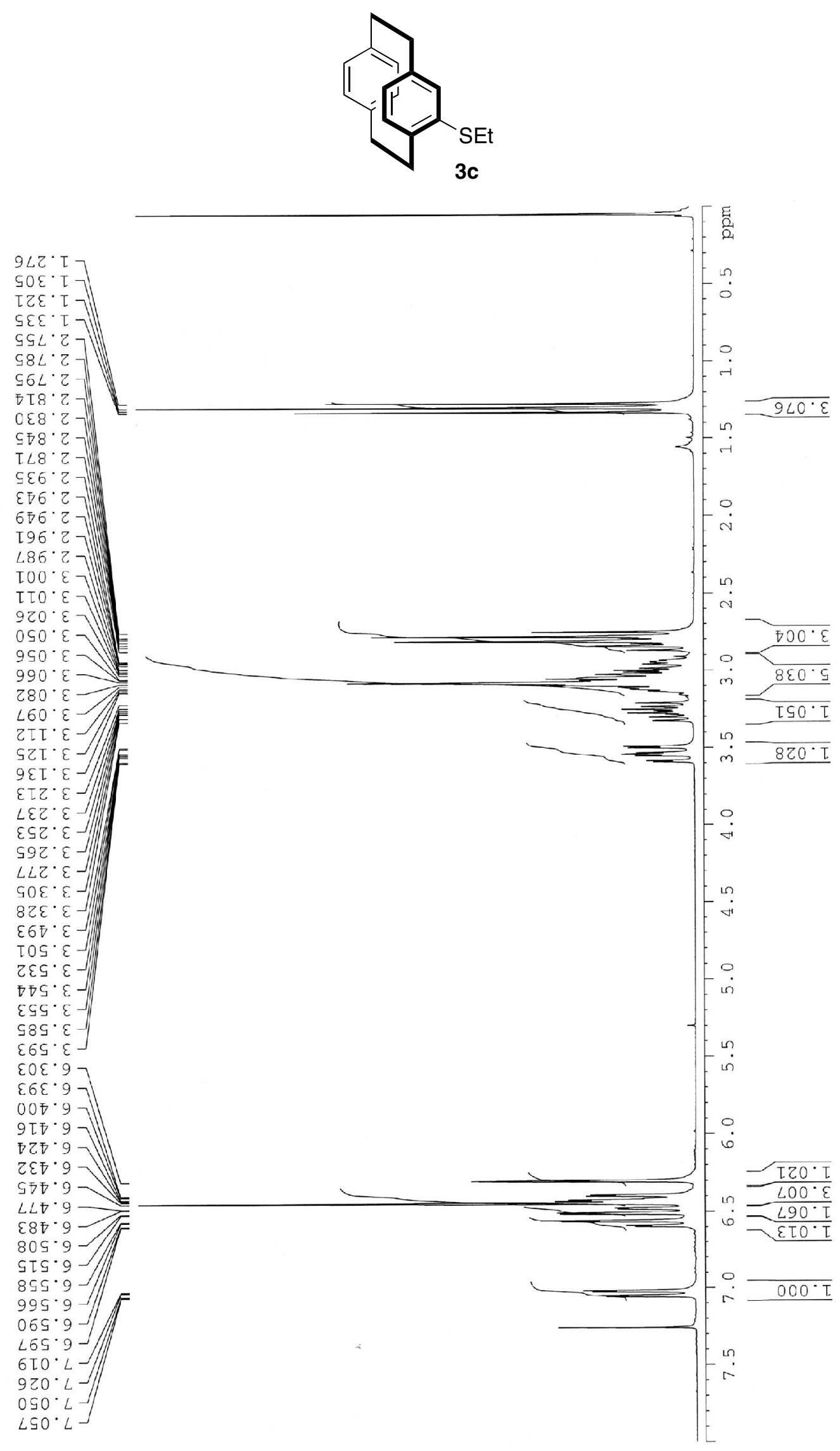


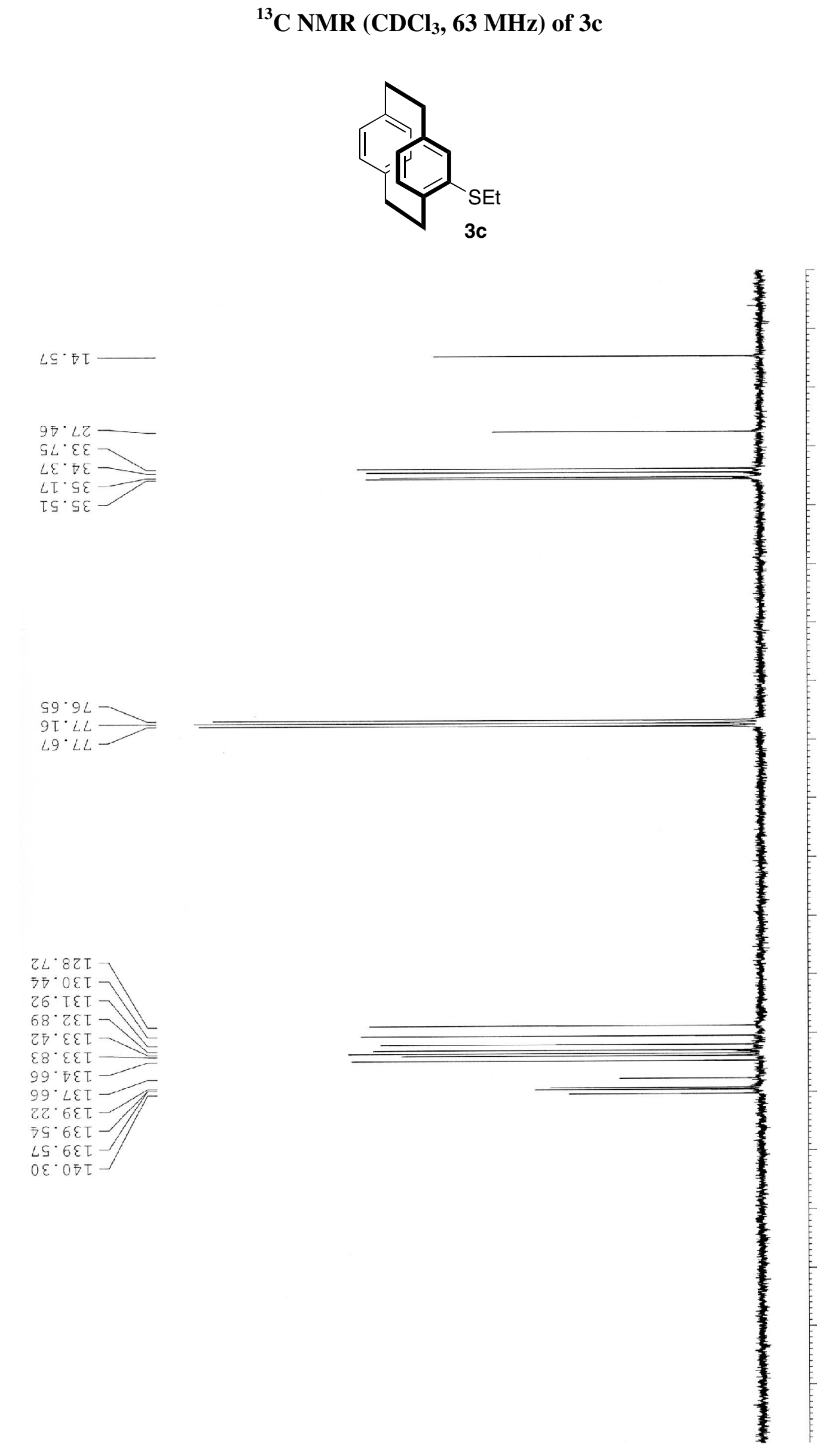


${ }^{1} \mathrm{H} \mathrm{NMR}\left(\mathrm{CDCl}_{3}, 250 \mathrm{MHz}\right)$ of 5

Mixture of diastereoisomers (3:2 ratio)
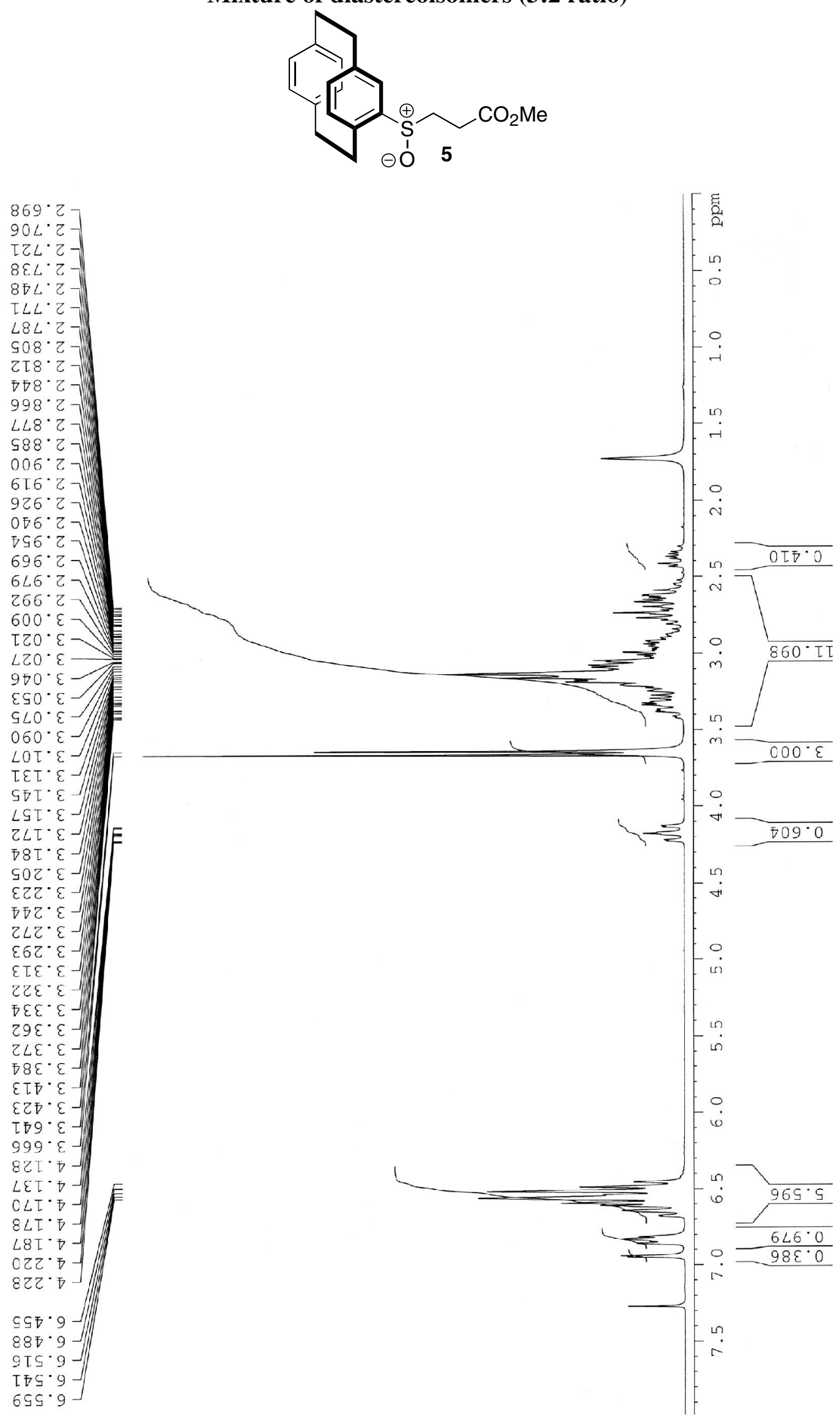
S31

${ }^{13} \mathrm{C} \mathrm{NMR}\left(\mathrm{CDCl}_{3}, 63 \mathrm{MHz}\right)$ of 5

Mixture of diastereoisomers (3:2 ratio)<smiles>CC(=O)CC[Sb](O)c1ccccc1CCc1ccc(C)cc1</smiles>
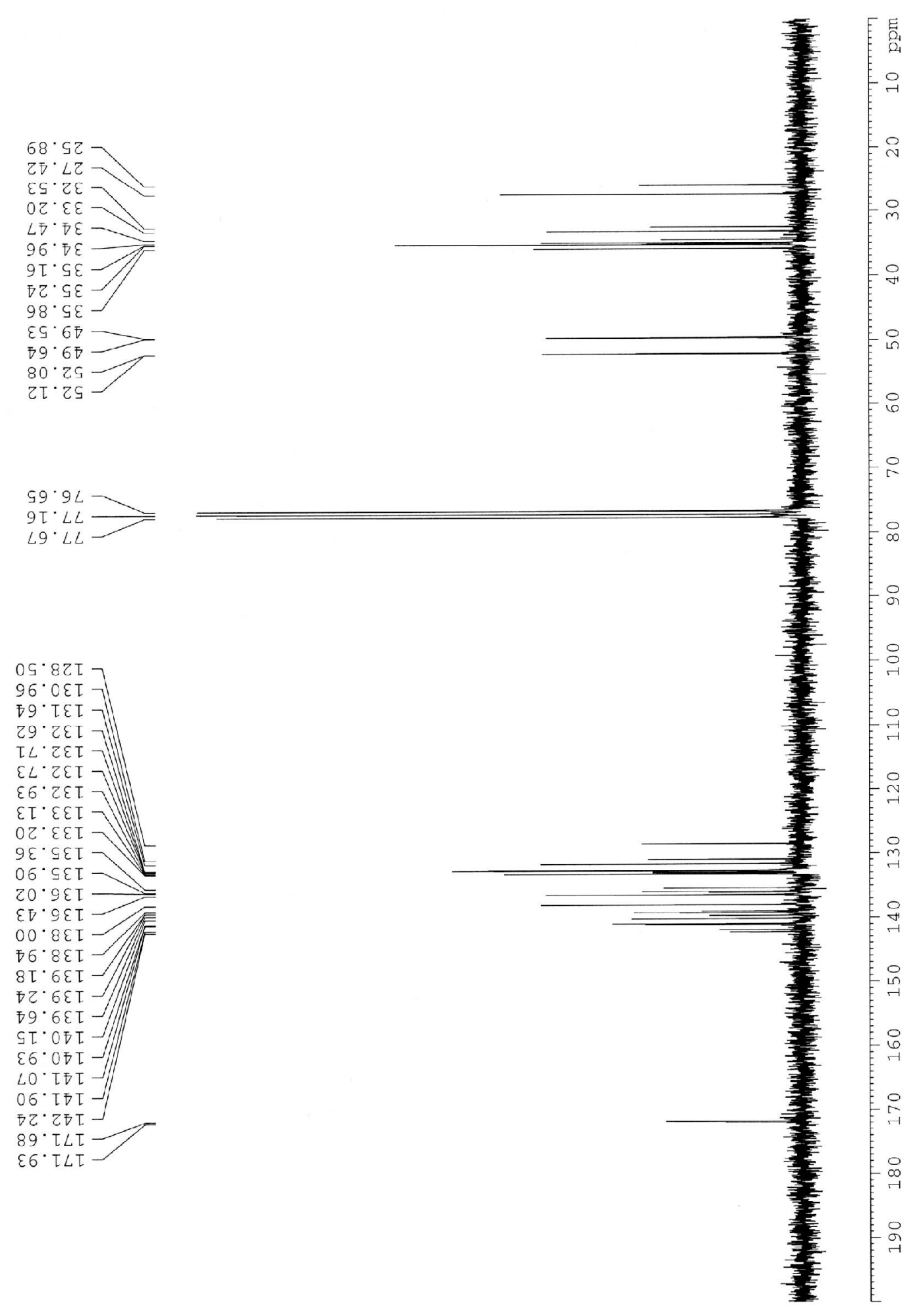
${ }^{1} \mathrm{H}$ NMR $\left(\mathrm{CDCl}_{3}, 250 \mathrm{MHz}\right)$ of 13
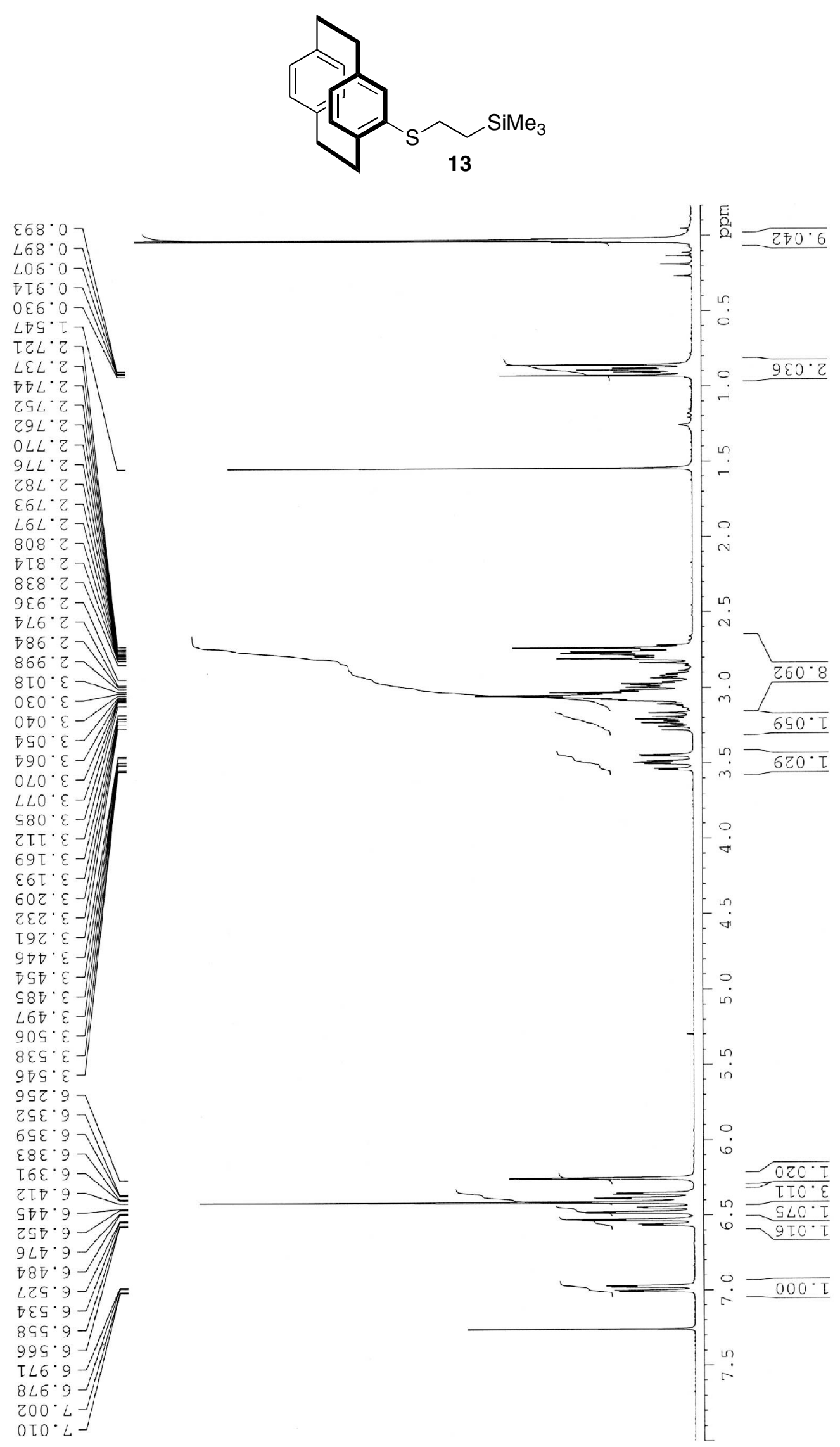


$$
1
$$


S34

ixture of diastereoisomers (7:3 ratio

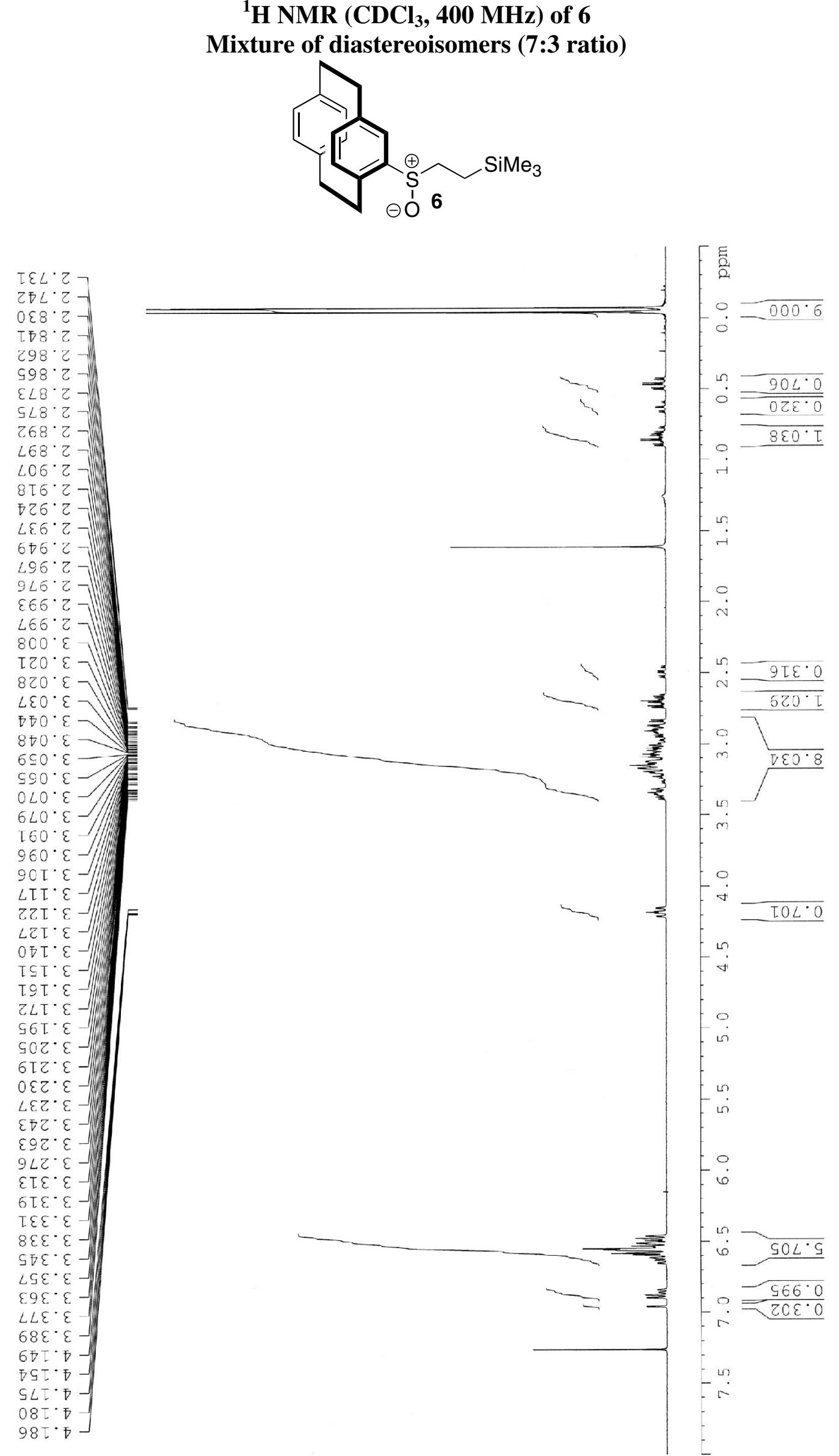


S35

${ }^{13} \mathrm{C} \mathrm{NMR}\left(\mathrm{CDCl}_{3}, 63 \mathrm{MHz}\right)$ of 6

Mixture of diastereoisomers (7:3 ratio)<smiles>C[SiH2]CC[Si](O)c1ccccc1CCc1ccc(C)cc1</smiles>

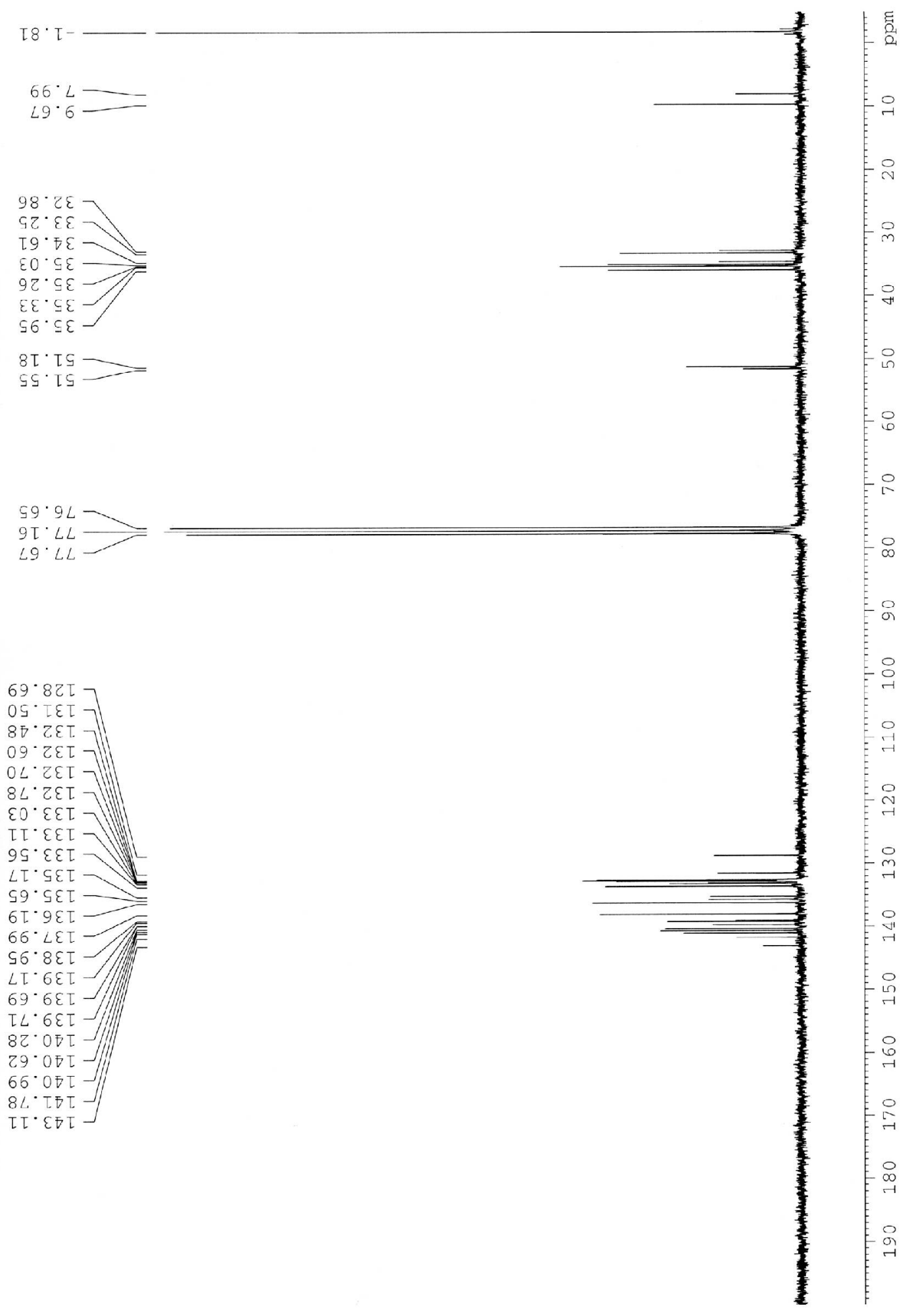


${ }^{1} \mathrm{H}$ NMR $\left(\mathrm{CDCl}_{3}, 250 \mathrm{MHz}\right)$ of 9a

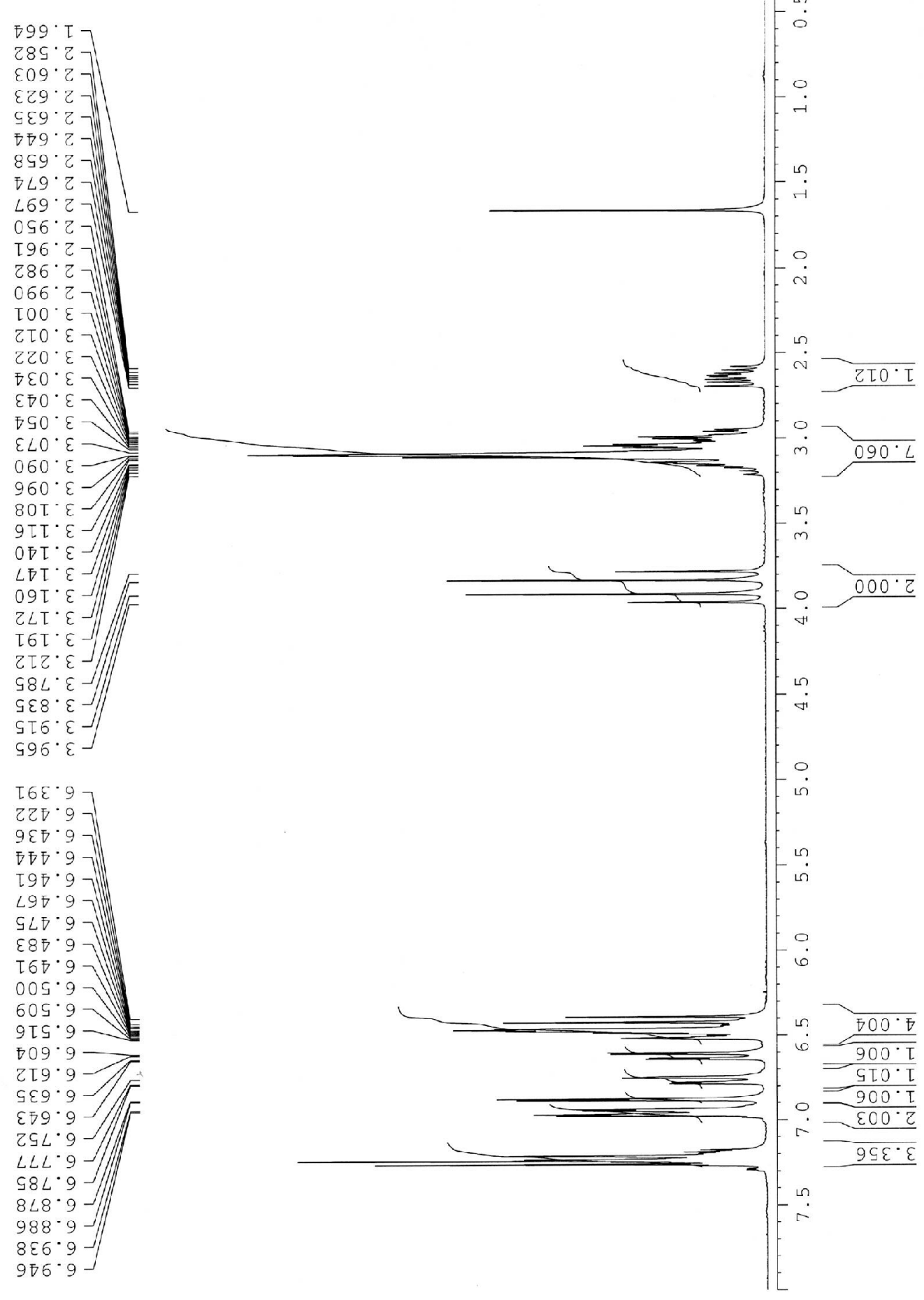


${ }^{13} \mathrm{C}$ NMR $\left(\mathrm{CDCl}_{3}, 63 \mathrm{MHz}\right)$ of $9 \mathrm{a}$
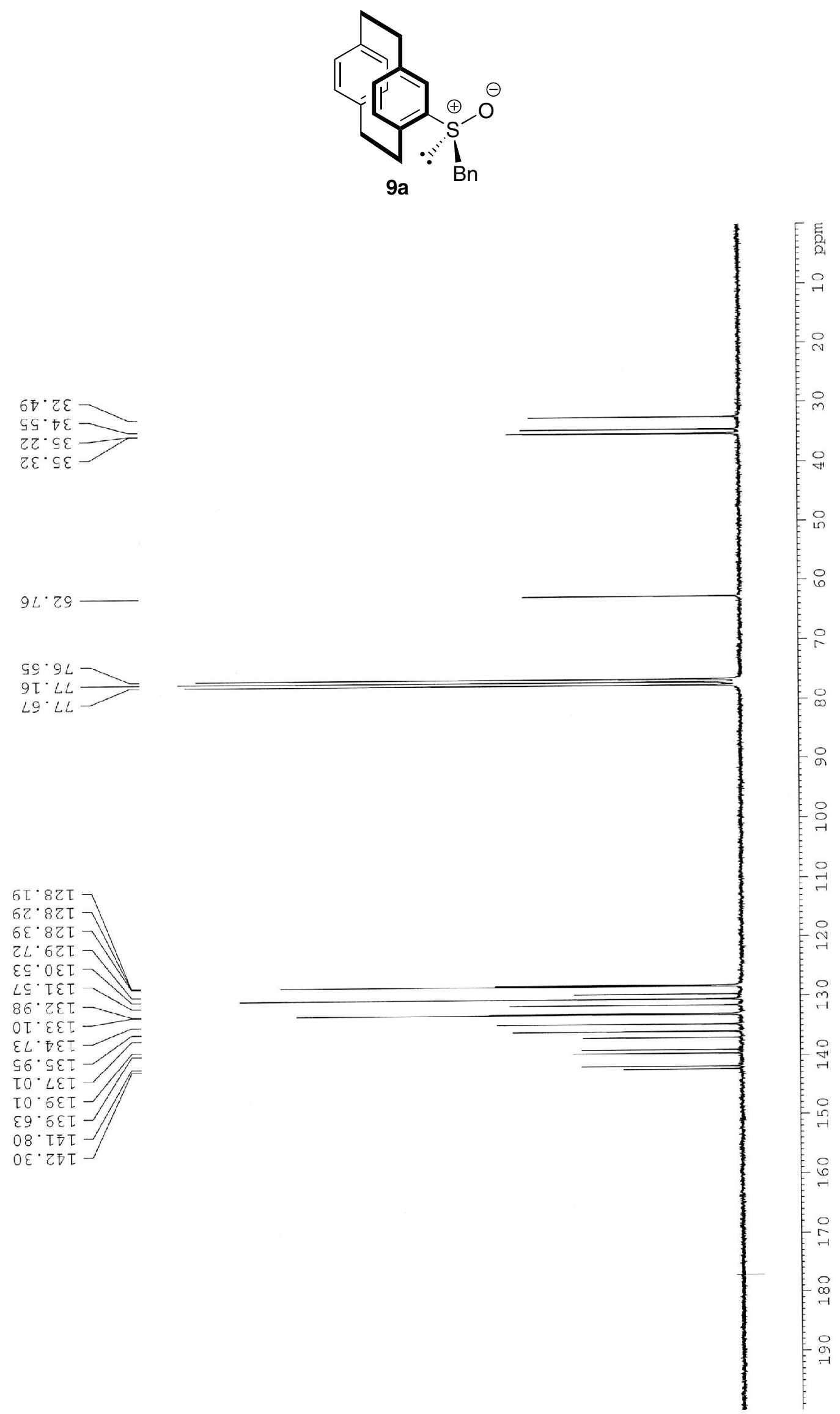
${ }^{1} \mathrm{H}$ NMR $\left(\mathrm{CDCl}_{3}, 250 \mathrm{MHz}\right)$ of $9 \mathrm{~b}$
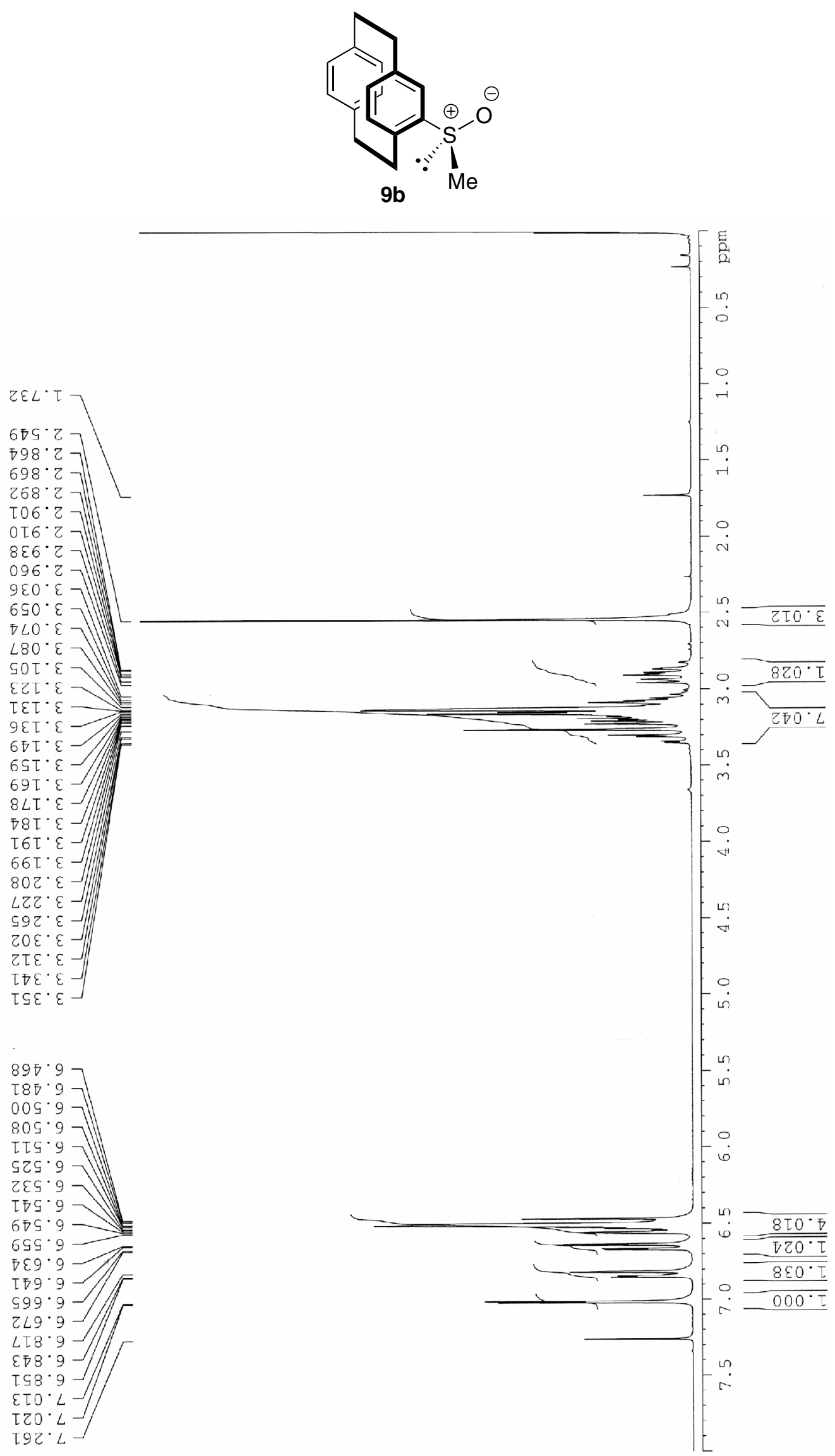
${ }^{13} \mathrm{C} \mathrm{NMR}\left(\mathrm{CDCl}_{3}, 63 \mathrm{MHz}\right)$ of $9 \mathrm{~b}$

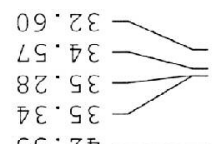

$G \mathcal{G}^{\circ} \mathrm{T}$

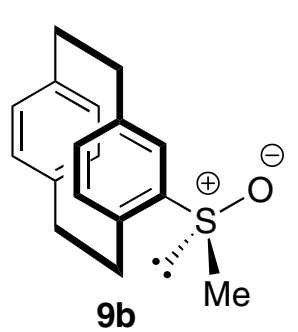

$59 \cdot 9 L-$

9
$\angle 9 \cdot L L$

$9 b$
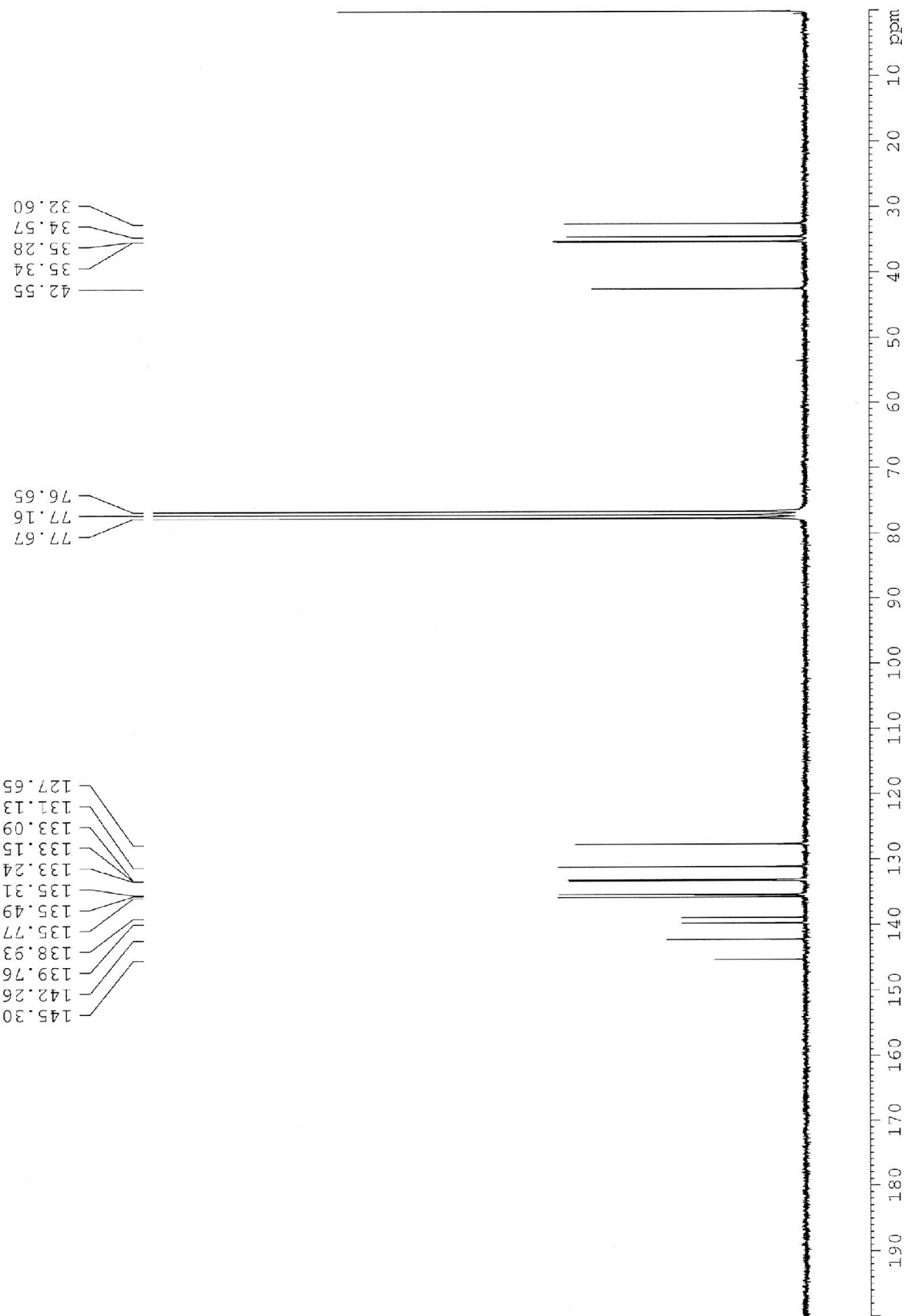
${ }^{1} \mathrm{H}$ NMR $\left(\mathrm{CDCl}_{3}, 250 \mathrm{MHz}\right)$ of $9 \mathrm{c}$
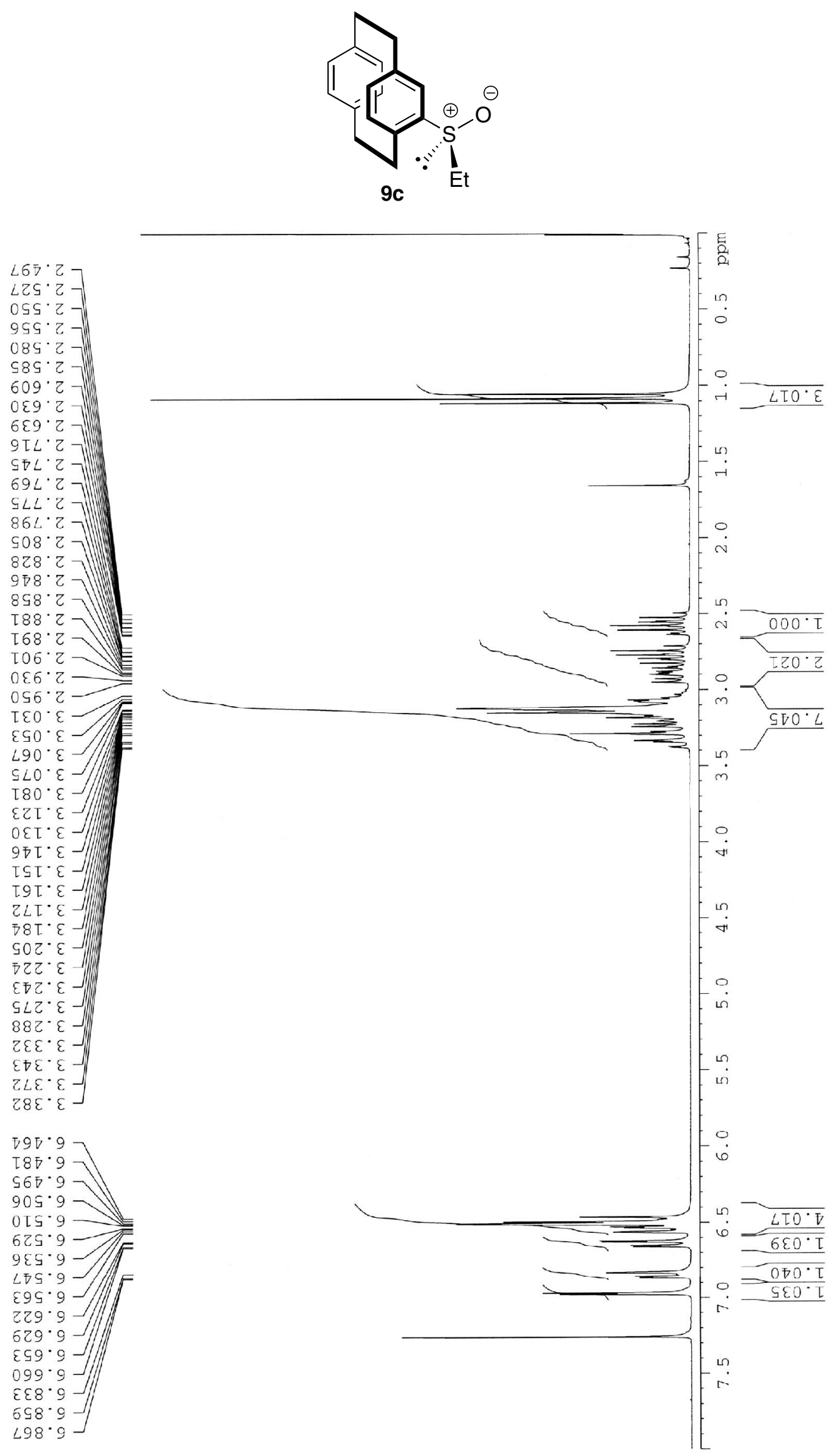


$$
\frac{\bar{x}}{11}
$$


${ }^{1} \mathrm{H}$ NMR $\left(\mathrm{CDCl}_{3}, 250 \mathrm{MHz}\right)$ of $10 \mathrm{a}$

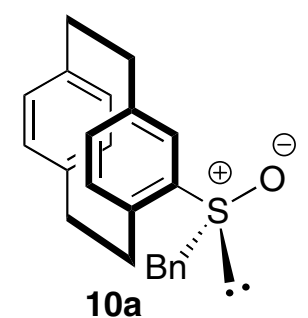

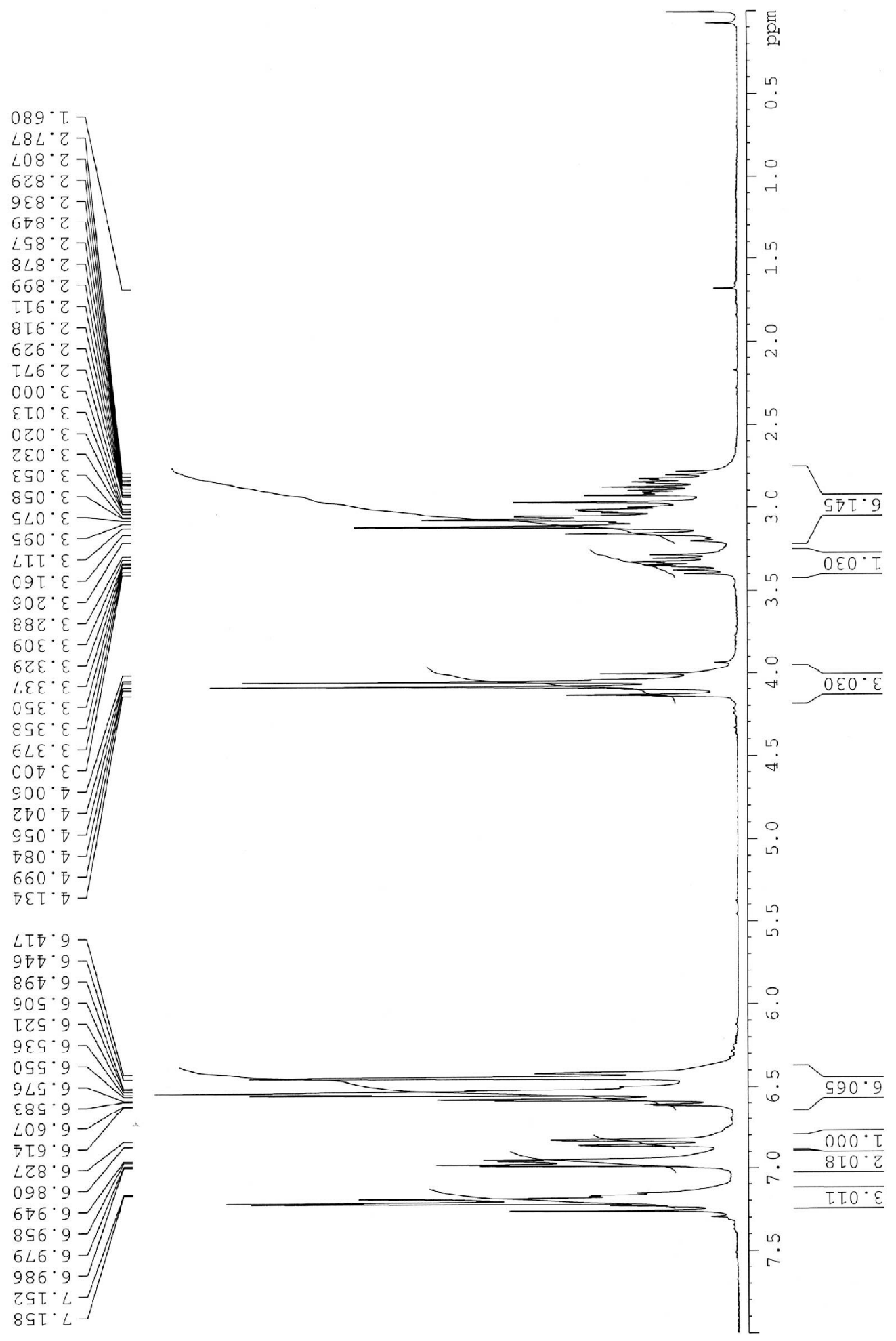


${ }^{13} \mathrm{C}$ NMR $\left(\mathrm{CDCl}_{3}, 63 \mathrm{MHz}\right)$ of $10 \mathrm{a}$

$00 \cdot \mathrm{s} \varepsilon$

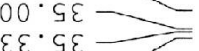

$66^{\circ} \mathrm{\varsigma} \varepsilon-$<smiles>CS([O-])(Br)c1ccccc1CCc1ccccc1</smiles>
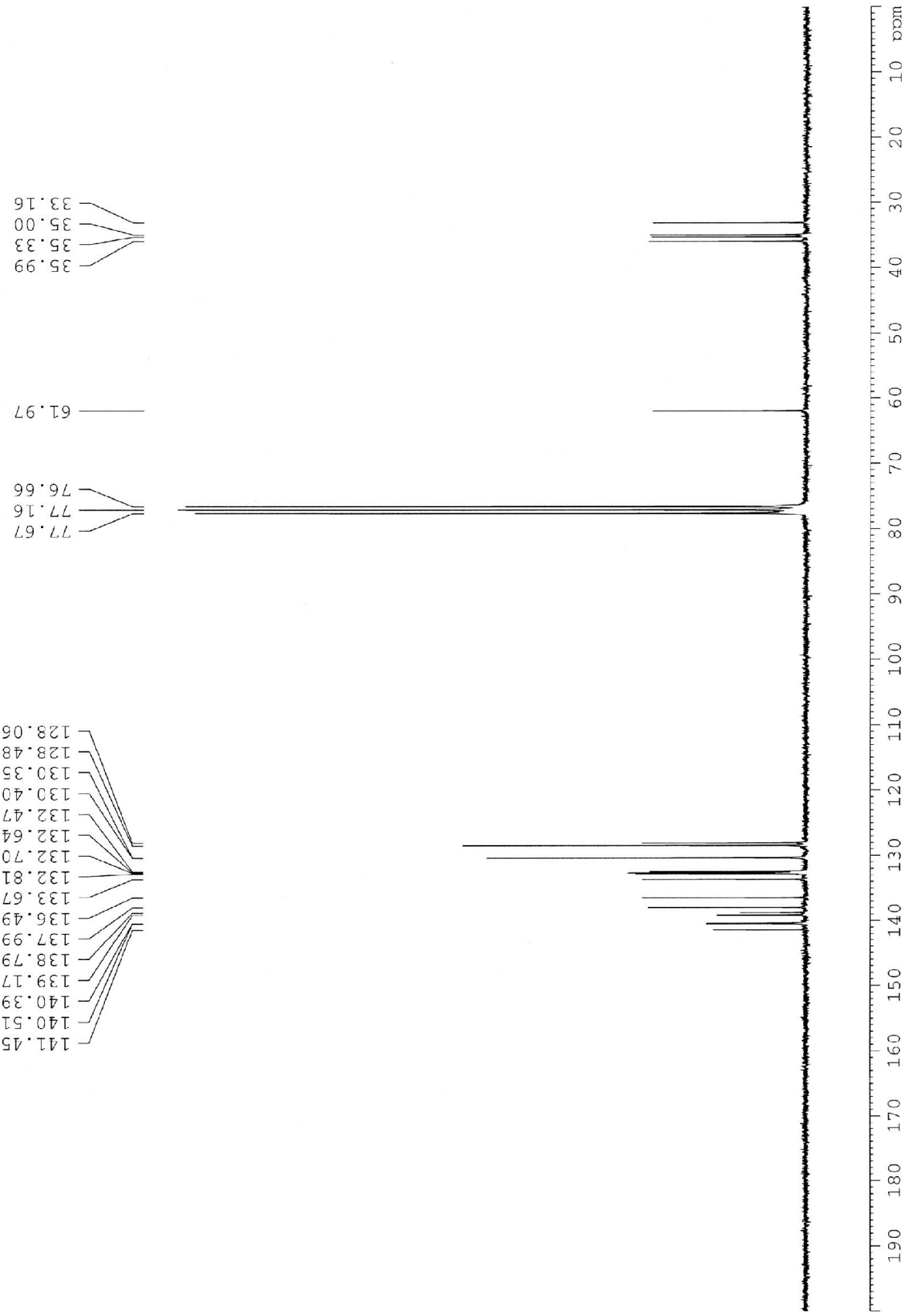
${ }^{1} \mathrm{H}$ NMR $\left(\mathrm{CDCl}_{3}, 250 \mathrm{MHz}\right)$ of $10 \mathrm{~b}$
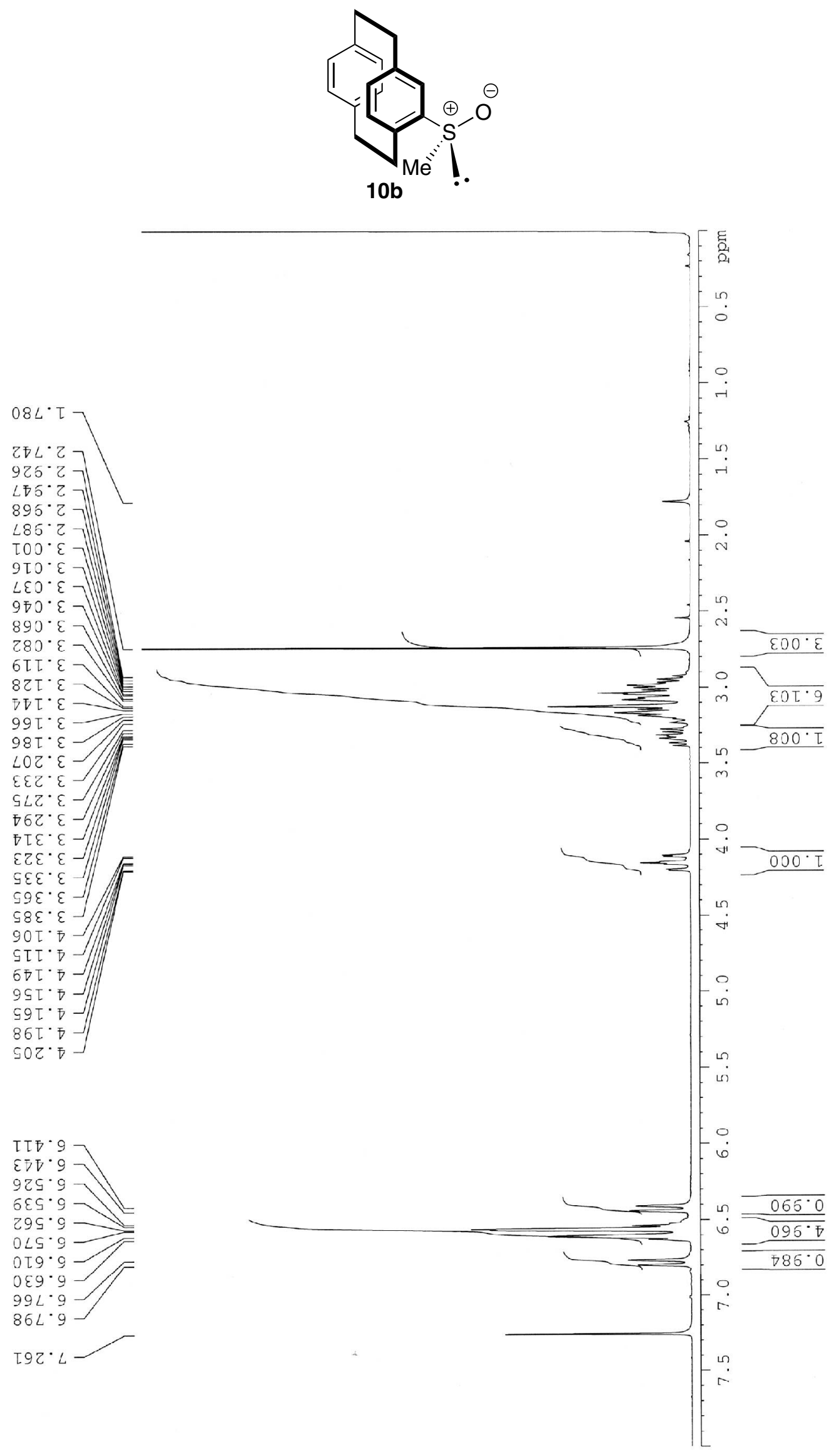
${ }^{13} \mathrm{C} \mathrm{NMR}\left(\mathrm{CDCl}_{3}, 63 \mathrm{MHz}\right)$ of $10 \mathrm{~b}$

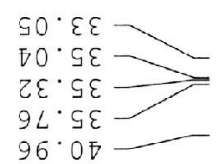<smiles>C[Si]([O])(O)C1=Cc2cc3ccc2C=CC(=C1)CC3</smiles>

$59 \cdot 94$

$9 \mathrm{I} \cdot \mathrm{LL} \longrightarrow$

$\angle E \cdot L L$

10b
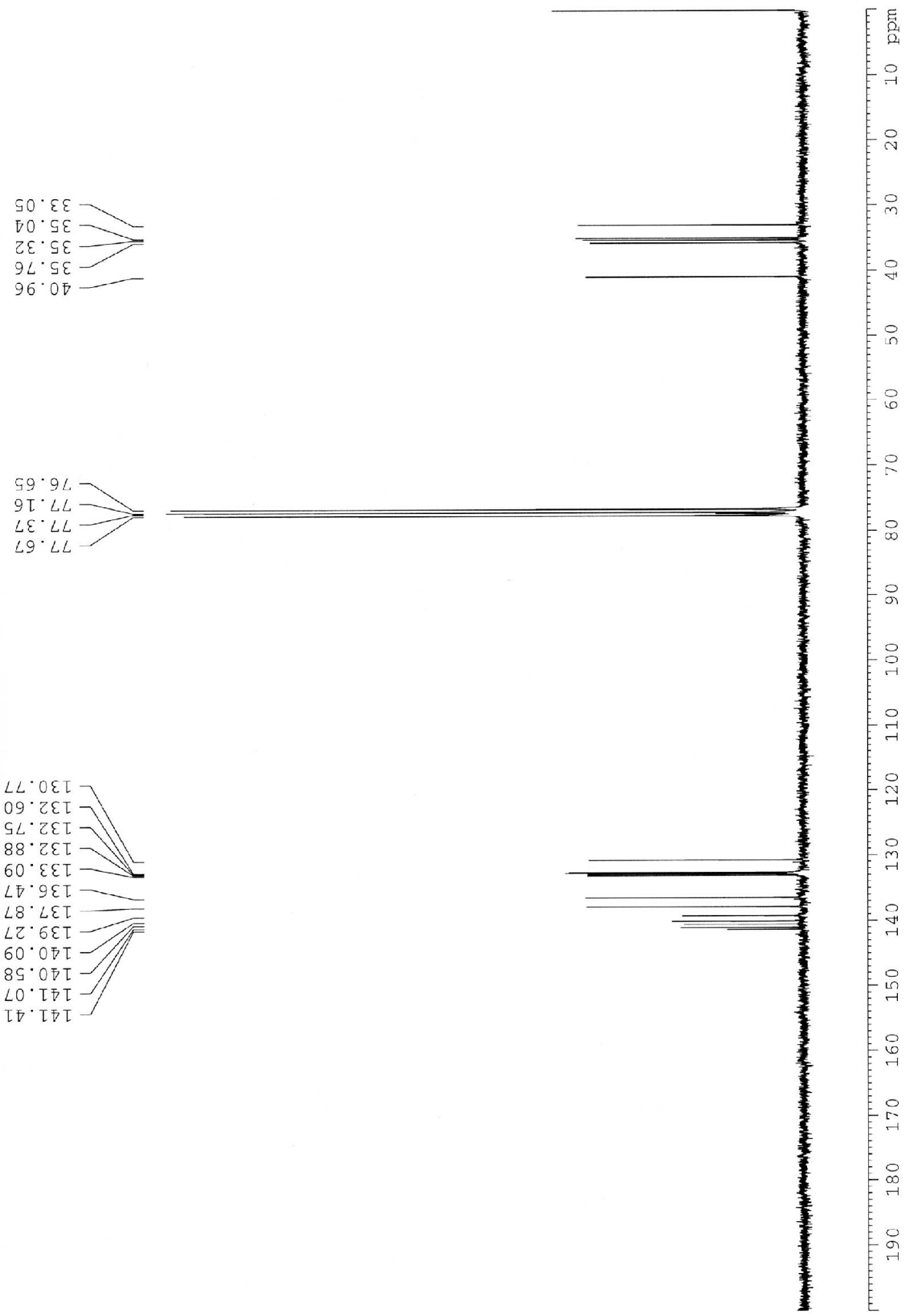
S46

${ }^{1} \mathrm{H}$ NMR $\left(\mathrm{CDCl}_{3}, 250 \mathrm{MHz}\right)$ of $10 \mathrm{c}$

[Mixture of 10c and 9c (81:19 ratio)]<smiles>CCCc1cc2c(cc1[Si](O)(O)CC)CCC2</smiles>

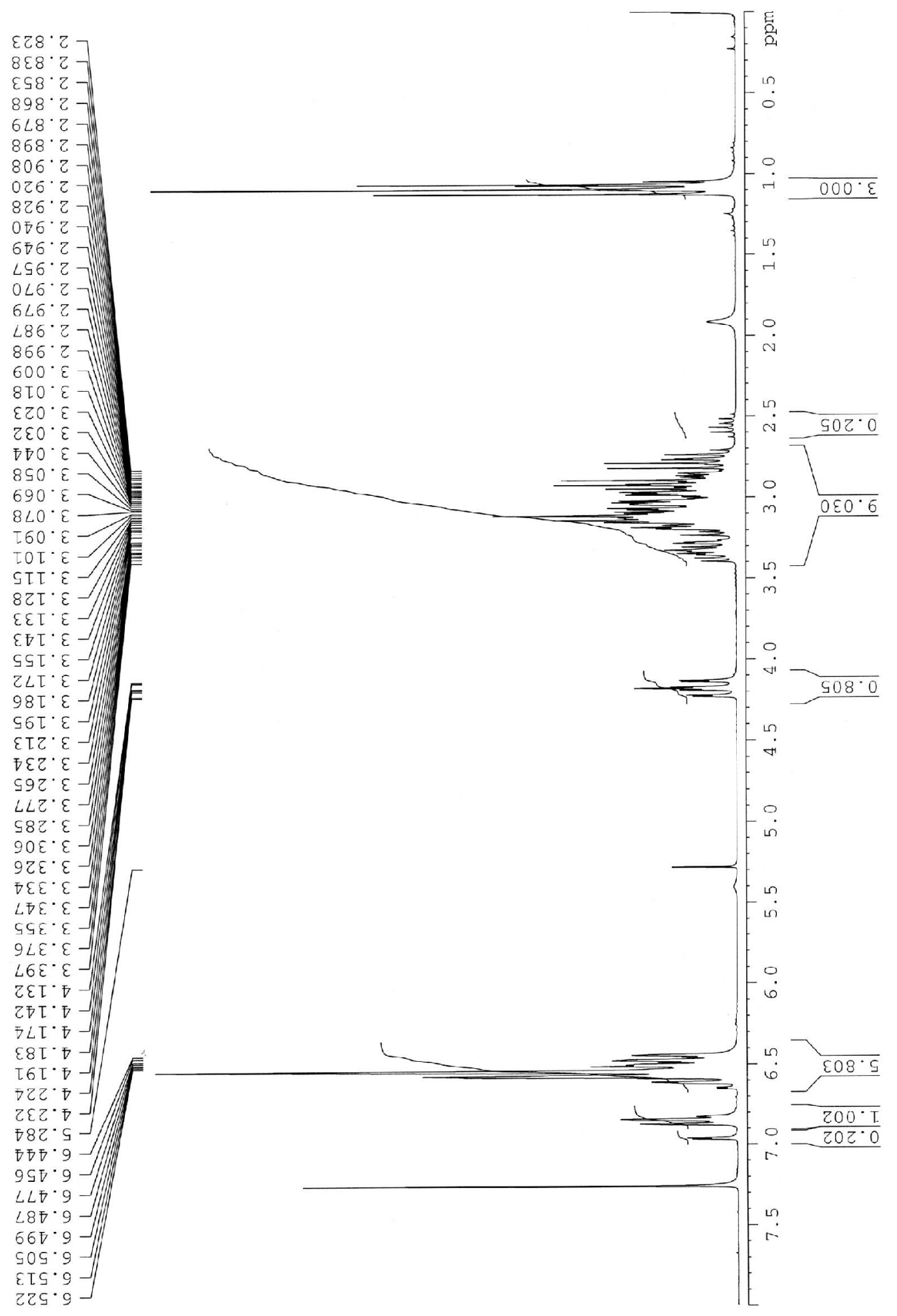


S47

${ }^{13} \mathrm{C} \mathrm{NMR}\left(\mathrm{CDCl}_{3}, 63 \mathrm{MHz}\right)$ of $10 \mathrm{c}$

[Mixture of 10c and 9c (81:19 ratio)]<smiles>CCCCc1cc2c(cc1[Si](O)(O)CC)CCC2</smiles>

$20 \cdot 9$
$80 \cdot 2$
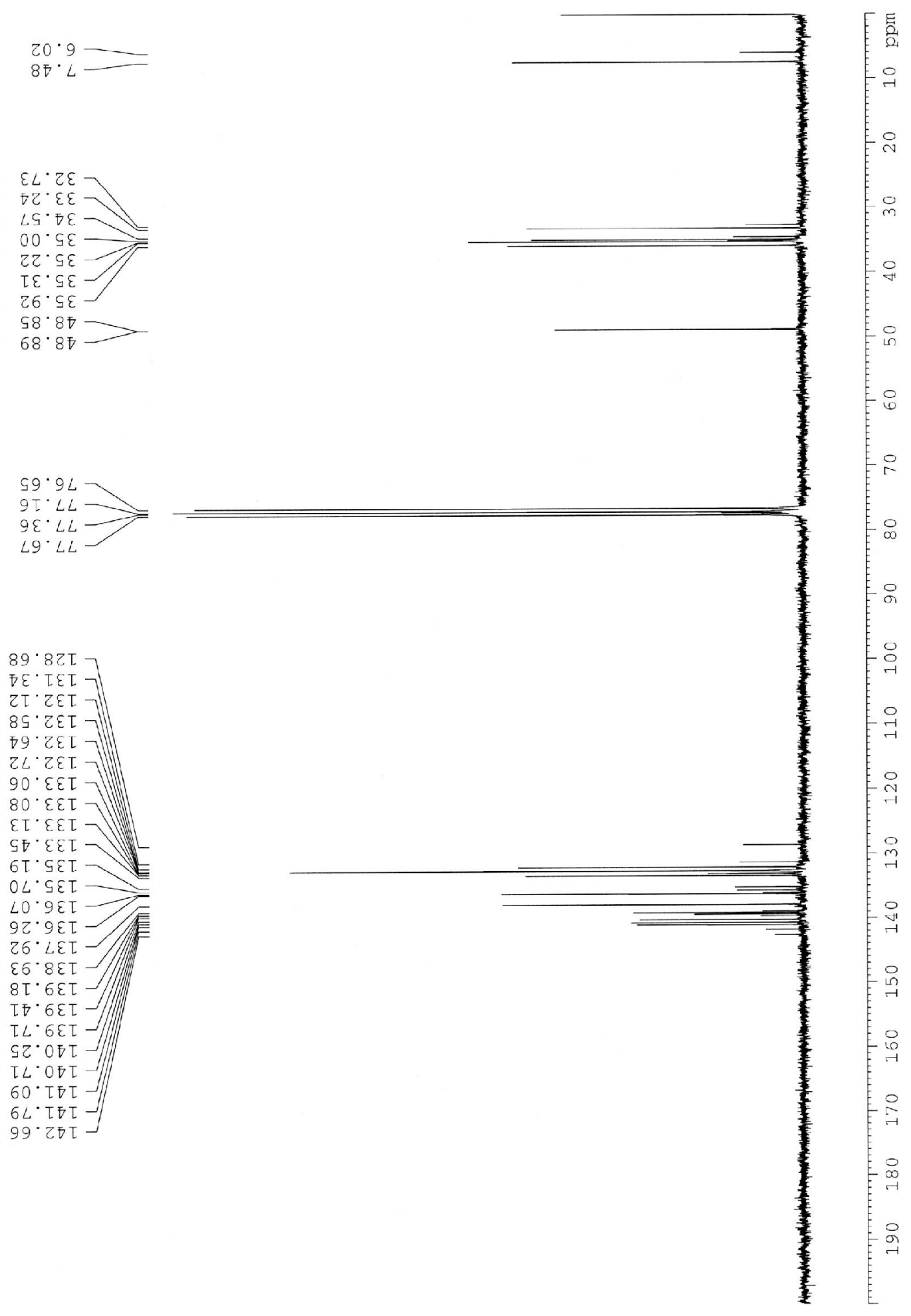